\title{
A Sparse Bayesian Imaging Technique for Efficient Recovery of Reservoir Channels with Time-lapse Seismic Measurements
}

\author{
Furrukh Sana $^{\dagger, 1}$, Fabio Ravanelli ${ }^{\S}$, Tareq Y. Al-Naffouri ${ }^{\dagger}$, I. Hoteit ${ }^{\dagger, \ddagger}$ \\ ${ }^{\dagger}$ Division of Computer, Electrical and Mathematical Sciences and Engineering, \\ ${ }^{\ddagger}$ Division of Physical Sciences and Engineering, \\ King Abdullah University of Science 83 Technology, 23955-9600 Thuwal, Saudi Arabia, \\ $\S$ Saudi Aramco, Saudi Arabia
}

\begin{abstract}
Subsurface reservoir flow channels are characterized by high permeability values and serve as preferred pathways for fluid propagation. Accurate estimation of their geophysical structures is thus of great importance for the oil industry. The Ensemble Kalman filter (EnKF) is a widely used statistical technique for estimating subsurface reservoir model parameters. However, accurate reconstruction of the subsurface geological features with the EnKF is challenging because of the limited measurements available from the wells and the smoothing effects imposed by the $\ell_{2}$-norm nature of its update step. A new EnKF scheme based on sparse domain representation was introduced by Sana et al. (2015) to incorporate useful prior structural information in the estimation process for efficient recovery of subsurface channels. In this paper; we extend this work in two ways: (i) investigate the effects of incorporat-
\end{abstract}

\footnotetext{
${ }^{1}$ Email: furrukh.sana@kaust.edu.sa
} 
ing time-lapse seismic data on the channel reconstruction, and (ii) explore a Bayesian sparse reconstruction algorithm with the potential ability to reduce the computational requirements. Numerical results suggest that the performance of the new sparse Bayesian based EnKF scheme is enhanced with the availability of seismic measurements, leading to further improvement in the recovery of flow channels structures. The sparse Bayesian approach further provides a computationally efficient framework for enforcing a sparse solution, especially with the possibility of using high sparsity rates through the inclusion of seismic data.

Keywords: Subsurface Channels Recovery, Reservoir History Matching, Ensemble Kalman Filter, Seismic Imaging, Sparsity, K-SVD, OMP, SABMP. 


\section{Introduction}

Accurate reconstruction of subsurface geological features is crucial in understanding the reservoir flow behavior $[1,2]$. Geological formations can be separated into two distinct groups, consisting of high permeability flow channels surrounded by low permeability rock formations $[3,4,5,6]$. The high permeability channels serve as preferred pathways for fluid propagation and aquifers may be conveniently placed on top of these channels to optimize reservoir productivity. Estimating the locations and the structures of these channels is therefore important for both forecasting the reservoir productivity and optimizing the future placements of the wells.

The Ensemble Kalman filter (EnKF) is a widely used Bayesian filtering technique for the estimation of subsurface reservoir parameters, such as

permeability $[4,7,8]$. However, recovering information on the subsurface geological structures with the standard EnKF is challenging. Because of its $\ell_{2}$-norm formulation, the EnKF imposes a smoothing effect on its estimates and often fails to preserve the channels structures and their connectivity [4]. The ill-posedness of the problem due to the limited number of measurements available from the wells further leads to multiple non-unique solutions and may result in large uncertainties in the estimated permeability field [9]. Moreover, the ability of the standard EnKF to incorporate prior information on the large number of possible subsurface geological features could be also largely limited by the ensemble size that can be used in practice. Jafarpour et al. [4], concluded that a large ensemble of 300 or more members is nec- 
essary for reasonable subsurface channels reconstruction. This, however, is a severely limiting factor considering the significant computational resources required for integrating each ensemble member forward with the reservoir simulator [10].

Recent works in the area of sparse signal reconstruction have provided efficient new frameworks for enhanced recovery of signals (or fields) exhibiting sparsity in some domain from a reduced number of observations by exploiting the prior information on signal sparsity $[11,12,13,14]$. For the problem under consideration, where the high permeability channels are expected to occupy only a limited portion of the reservoir, the subsurface field mostly comprises smooth (or piecewise smooth) features exhibiting spatial continuity and long range correlations. These features enable the formulation of the channel recovery problem as a sparse field recovery problem $[11,15,12,16]$. Selection of a suitable sparse domain that incorporates and preserves structural information in the estimation process can further help in recovering subsurface geological structures $[3,17,18,16]$.

Generic basis functions, such as Discrete Cosine Transforms (DCT) and the Wavelet transforms, have been considered to help improving channels recovery within an EnKF framework [19, 20]. Such schemes exploit sparse transforms to represent the permeability field using a much smaller number of variables. However, these basis functions only help achieving performances comparable to a standard spatial domain EnKF, while improving the illposedness of the problem [19, 20]. Principal Component Analysis (PCA) 
based geologic basis functions were also investigated in [18] under a hybrid parametrization framework. However, the PCA basis functions did not prove useful for recovering complex geological structures, such as meandering flow channels [18]. A sparse Bayesian estimation approach was presented in [21], where the Gaussian prior for the gradient is replaced by a Laplace prior and is combined with DCT transform domain parameterization. This probabilistic approach is derived from a machine learning algorithm for linear inverse problems and avoids the need for regularization. A similar scheme was adopted in [22] where it was combined with an expectation-maximization (EM) algorithm for maximizing the marginal distribution of the estimated parameters. The scheme was shown to be effective when discrete geologic structures with relatively homogeneous properties predominate the subsurface.

Iterative schemes were also proposed in $[3,17,23]$ in which sparse geologic dictionaries were learned by training over a large set of geological structures, varying in shapes, numbers, and locations. These can potentially lead to enhanced recovery of complex geological structures. However these, as well as those in $[11,18,16]$, require implementing the adjoint model for efficient computation of the gradients of the objective function. This can be a particularly challenging and demanding process, especially when dealing with realistic large scale reservoir simulations. [24, 25].

Greedy sparse reconstruction algorithms offer an efficient framework for enforcing sparsity constraints within an estimation process [26, 27]. A new scheme was introduced in [28] to enhance the recovery of the subsurface ge- 
ological features, combining the EnKF with the Orthogonal Matching Pursuit (OMP) algorithm. The proposed Sparse Geological Structures Domain (SGSD)-EnKF algorithm transforms the EnKF estimation of the subsurface geological structures to a specifically constructed sparse domain learned over a large training set and was shown to provide significant improvement in recovering the channels over the standard EnKF. A more detailed discussion of the SGSD-EnKF scheme followed in [29], providing comprehensive analysis of the channels recovery performance against various factors such as the ensemble size, sparsity rate ${ }^{2}$, wells configuration, etc.. It was however observed that the number of basis elements with non-zero coefficients that could be used to improve the channel reconstruction was limited by the amount of observations available from the wells. The production data from the wells alone did not provide sufficient information to enable using more than $10 \%$ of the basis elements (i.e. a sparsity rate of 10\%) in the estimation process, with higher sparsity rates resulting in degraded performances [29].

The inclusion of time-lapse seismic data, also known as 4D seismic, in the EnKF based history matching process may potentially help improving the understanding of the subsurface structures and reservoir connectivity as characterized by the permeability field [30]. A seismic survey images the subsurface by gathering elastic wave information. Seismic waves are usually generated at the surface and propagated through the medium as they travel.

\footnotetext{
${ }^{2}$ The sparsity rate is defined as $p=\frac{\text { number of non-zero coefficients }}{\text { total length of the estimated vector }} \times 100$
} 
They are reflected along the interfaces of the subsurface layers with different acoustic impedance. The returning waves are then recorded by the sensors at the surface, providing critical information about the medium [31].

A single snapshot of the reservoir based on a 3D seismic acquisition is not enough to uniquely separate geological and fluid flow components of the reservoir model. 4D seismic consists of a set of inverted images of seismic attributes obtained at different times and subtracted from each other. The time-lapse signal changes as the reservoir conditions change from oilsaturated to water saturated [32]. The time-difference can hence be used to infer changes in the dynamic properties of the reservoir and to separate the time invariant component (i.e. the geological model) from the dynamic fluid flow component, which should provide information to help recovering the geological structures [30,33]. The first successful use of $4 \mathrm{D}$ seismic under an EnKF framework was presented by Skjervheim et al. in [34] and more recently gained momentum in helping to monitor the fluid flow movement in the reservoir [35, 36, 37, 38].

Time-lapse seismic data is expected to enhance the estimation process with needed information from the additional observations, which should also enable effective use of more basis elements to further improve the recovery of the channels structures. While the reconstruction performance is expected to improve at higher sparsity rates, the OMP algorithm may not be the best choice for the sparse transformation step in the SGSD-EnKF in terms of computational cost. The complexity of the OMP algorithm is anticipated 
to increase linearly with increasing sparsity rates and necessitates the use of a more efficient algorithm that can provide improved reconstruction over the standard EnKF while maintaining the added computational costs within acceptable range.

In this paper; we extend the work of [28] and [29] to investigate the effects of time-lapse seismic data on the channel reconstruction performance, particularly focusing on overcoming the limitation on the sparsity rate as observed in [29]. We explore a Bayesian sparse reconstruction algorithm to deal with the expected increase in computational costs at higher sparsity rates with the OMP algorithm [29]. Moreover, we present a slightly modified SGSD-EnKF algorithm which now includes a more strict sparsity constraint within the EnKF update step. We analyze the estimation quality and computational complexities of the improved SGSD-EnKF algorithm against the ensemble size and sparsity rate, the two factors that considerably affect the computational burden. Our numerical results suggest that the inclusion of seismic data greatly helps in leveraging the large amount of prior information available through the use of more basis elements, leading to even further improved reservoir channels reconstruction with the SGSD-EnKF. The Bayesian sparse reconstruction approach further provides a viable alternative to the OMP algorithm, demonstrating a significant advantage in terms of computational complexity while delivering a comparable reconstruction quality.

The paper is organized as follows. Section 2 provides a brief overview of the components of the sparse estimation framework and the improved ver- 
sion of the SGSD-EnKF algorithm originally proposed in [28, 29]. Section 3 discusses the experimental setup coupling a two-phase subsurface flow model with an EnKF, and describes the simulation parameters. Section 4 presents and analyzes the results of the numerical experiments. The paper concludes with a general discussion and summary of the main results in Section 5 .

\section{Sparse Ensemble Estimation Framework}

\subsection{The Ensemble Kalman Filter (EnKF)}

The EnKF is a popular Monte Carlo variant of the standard Kalman filter (KF) and has been successfully applied in a variety of history matching problems $[8,39,36,40]$. As the $\mathrm{KF}$, the EnKF estimation process is based on forecast and analysis cycles but differs in the way the first two moments of the system state are represented by an ensemble of state vectors, approximating the KF estimate and associated error covariance matrix by the ensemble sample mean and covariance. This formulation allows to significantly reduce the computational complexity when dealing with large scale systems [7]. The ensemble formulation further helps dealing with the nonlinearity of the problem, which allows to avoid the need for computing the Jacobian matrices as required in the Extended Kalman filter (EKF). The formulation of the problem is based on a state-space model of the form

$$
\begin{gathered}
\mathbf{x}_{t+1}=\mathbf{M}_{t}\left(\mathbf{x}_{t}\right)+\boldsymbol{\eta}_{t}, \\
\mathbf{y}_{t}=\mathbf{h}_{t}\left(\mathbf{x}_{t}\right)+\boldsymbol{\epsilon}_{t},
\end{gathered}
$$


where $\mathbf{M}_{t}$ represents the reservoir simulator, the state vector $\mathbf{x}_{t}=\left[\mathbf{k}_{t}, \boldsymbol{\phi}_{t}, \mathbf{p}_{t}, \mathbf{s}_{t}\right]$ is composed of the static parameters, permeability $\mathbf{k}$ and porosity $\boldsymbol{\phi}$, and dynamic variables, pressure $\mathbf{p}$ and saturation $\mathbf{s}$, defined over the entire grid space at time $t$, and $\boldsymbol{\eta}_{t}$ a stochastic term representing the model error. The observation $\mathbf{y}_{t}$ is related to the state via an (nonlinear) observation operator $\mathbf{h}_{t}$, perturbed by a random noise $\boldsymbol{\epsilon}_{t}$. Here, the observation is composed of the production data, including the flux, the bore hole pressure (bhp), and the water cut ratio (wct), and the time-lapse seismic data. Both $\boldsymbol{\eta}_{t}$ and $\boldsymbol{\epsilon}_{t}$ are assumed independent and normally distributed of mean zero and covariance matrices $\mathbf{Q}_{t}$ and $\mathbf{R}_{t}$, respectively.

Starting from a given forecast ensemble $\mathbf{X}_{t}^{f}=\left[\mathbf{x}_{1, t}^{f}, \ldots, \mathbf{x}_{N_{e}, t}^{f}\right]$ of $N_{e}$ members at time step $t$, with $\mathbf{x}_{i, t}^{f}$ denoting the $i$-th forecast member, the forecast covariance anomaly is defined as $\mathbf{A}_{t}=\mathbf{X}_{t}^{f}-\frac{1}{N_{e}}\left(\sum_{i=1}^{N_{e}} \mathbf{x}_{i, t}^{f}\right) \mathbf{e}_{1 \times N_{e}}$, with $\mathbf{e}_{1 \times N_{e}}$ denoting the vector with ones as elements, and the ensemble observation matrix $\left[\mathbf{H}_{t}\right]_{:, i}=\mathbf{h}_{t}\left(\mathbf{x}_{i, t}^{f}\right)-\frac{1}{N_{e}} \sum_{j=1}^{N_{e}} \mathbf{h}_{t}\left(\mathbf{x}_{j, t}^{f}\right)$. Using the data matrix $\mathbf{D}_{t}$ whose columns are the observations perturbed with noise sampled from the distribution of $\boldsymbol{\epsilon}_{t}$, and the corresponding ensemble covariance matrix $\tilde{\mathbf{R}}_{t}$, the EnKF update step uses the observation to compute the analysis ensemble $\mathbf{X}_{t}^{a}$ from the forecast ensemble $\mathbf{X}_{t}^{f}$ based on the Kalman filter update step as

$$
\mathbf{X}_{t}^{a}=\mathbf{X}_{t}^{f}+\frac{1}{N_{e}-1} \mathbf{A}_{t} \mathbf{H}_{t}^{T}\left(\frac{1}{N_{e}-1} \mathbf{H}_{t} \mathbf{H}_{t}^{T}+\tilde{\mathbf{R}}_{t}\right)^{-1}\left(\mathbf{D}_{t}-\mathbf{h}_{t}\left(\mathbf{X}_{t}^{f}\right)\right)
$$

The resulting analysis ensemble members are then integrated forward 
in time with the reservoir simulator using (1) for forecasting, before a new update step with the new incoming observation takes place. The estimate at any time $t$ is taken as the mean of the analysis ensemble. The EnKF reservoir history matching process is illustrated in Figure 3.

In the context of Bayesian formulation of the filtering problem, the EnKF can be considered as a hybrid scheme relying on a Monte Carlo approach to propagate the probability distribution of the state vector in time for forecasting, and assuming Gaussian distributions at the update step [41]. This reduces the Bayesian inversion step with the observation to a linear Kalman update step. Despite the fact that the EnKF updates are only based on means and covariances and that these covariances are generally computed from finite size ensembles, the EnKF was shown to perform very well for a variety of history matching problems [8]. The reader may refer to [8] and [42] for further details about the EnKF.

\subsection{The SGSD-EnKF Algorithm}

Recent works in the area of sparse transform domain signal reconstruction provide many tools to overcome the limitations of the EnKF in recovering subsurface channels. Identification of a suitable transform domain in which the signal, or field, can be modeled as sparse is critical for fully leveraging the benefits of any sparse parametrization framework. An efficient and robust sparse basis for representing the permeability ensemble can be constructed using dictionary learning algorithms [3]. The idea is then to incorporate 
useful prior information on a large number of different possible subsurface geological structures into the estimation process.

Dictionary learning algorithms allow constructing a sparse domain by learning over a large collection of training data. Given $N_{t}$ number of training signals in a set $\mathbf{Y}$, a dictionary learning algorithm constructs a dictionary $\Psi$ by finding the basis elements such that their sparse linear combinations can represent any of the signals in the training set $\mathbf{Y}$. The set of coefficients used for this sparse linear combination $\mathbf{X}$ represents the signals in the new sparse domain. Dictionary learning algorithms solve the optimization problem [43]

$$
\min _{\mathbf{X}, \mathbf{\Psi}}\left\{\|\mathbf{Y}-\mathbf{\Psi} \mathbf{X}\|_{2}^{2}\right\} \text { s.t. }\left\|\mathbf{x}_{i}\right\|_{0} \leq \mathbf{T}_{0} \forall i=1, \ldots, N_{t},
$$

where $\left\|\mathbf{x}_{i}\right\|_{0}$ denotes the $\ell_{0}$-norm and $\mathbf{T}_{0}$ represents the desired number of nonzero entries for each of the signals in $\mathbf{X}$, i.e. the sparsity rate. While a large value of $\mathbf{T}_{0}$ may help reducing the representation error, it also increases the computational complexity of the problem. Choosing a value for $\mathbf{T}_{0}$ should therefore balance between the computational complexity and the representation error. The optimization problem above is non-convex and NP-hard, as it attempts to minimize the representation error for the signals in $\mathbf{Y}$ by simultaneously adjusting the basis elements in $\mathbf{\Psi}$ and at the same time finding their corresponding coefficients in $\mathbf{X}$.

K-SVD is a dictionary learning algorithm that recursively solves the problem defined in equation (4) in two-steps [43]. In the first step, the basis 
elements of the dictionary are fixed, while their weights are calculated such that the representation error is minimized. The algorithm next attempts to further reduce the error by adjusting the basis elements themselves based on the weights computed in the previous step. For further details about the K-SVD algorithm, the reader may refer to [43]. [28, 29] showed that a large set of realizations, each depicting a different subsurface geological field structure, can be used to train the K-SVD algorithm in order to generate a sparse basis to use within an EnKF history matching framework. The proposed Sparse Geological Structures Domain (SGSD)-EnKF scheme in [28, 29] transforms the ensemble members representing the permeability field into the sparse domain defined by this new basis. The coefficients used for the sparse transformation represent the ensemble members in the new sparse domain and are then used in the EnKF update process. The ensemble members are then transformed back to the spatial domain before conducting the forecast step.

A slightly modified version of the SGSD-EnKF algorithm is presented in Algorithm 1 where we re-write the sparse transformation step (step 2) in its most general form. We also impose a constraint on the number of non-zero basis elements weights after the EnKF update. This constraint helps preserving the same sparsity rate throughout the history matching process. It is important to realize that the sparse transformation step (step 2) is different from the optimization problem for dictionary learning discussed above as it only attempts to represent the signal $\mathbf{K}_{t}^{f}$ by finding the coefficients $\boldsymbol{\kappa}_{t}^{f}$ 
for the basis elements of the fixed dictionary $\boldsymbol{\Psi}$ computed offline using the K-SVD. The original SGSD-EnKF algorithm proposed in [28, 29] uses the OMP algorithm [26] to compute the solution to this step. Despite the fact that the OMP algorithm is expected to provide improved reconstruction with

increased sparsity rates (made possible with the inclusion of seismic data), the anticipated linear increase in the computational complexity necessitates the use of a more efficient algorithm that can provide comparable reconstruction quality while scaling the computational complexity much slowly. In the next section, we present the Support Agnostic Bayesian Matching Pursuit (SABMP) algorithm that can potentially help us achieve both of these objectives.

\subsection{The $S A B M P$ Algorithm}

The Orthogonal Matching Pursuit (OMP) algorithm uses only the information on signal sparsity to compute the sparse solution. The SABMP algorithm, in contrast, also utilizes the Bayesian statistics of the signal and noise, allowing to achieve effective computations at much faster rates [27]. The SABMP algorithm does not make any assumption on the distribution of the signal, making it particularly suitable for our application. The MMSE estimate in the SABMP algorithm is given by the expression

$$
\hat{\mathbf{x}}_{m m s e} \triangleq E[\mathbf{x} \mid \mathbf{y}]=\sum_{S} p(S \mid \mathbf{y}) E[\mathbf{x} \mid \mathbf{y}, S]
$$


Algorithm 1 Sparse Geological Structures Domain EnKF (SGSD-EnKF) Algorithm

1. Input: Forward permeability ensemble $\mathbf{K}_{t}^{f}, n \times d$ dictionary $\mathbf{\Psi}$ (generated once using K-SVD), observation data $\mathbf{y}_{t}$, sparsity level $p$.

2. Transform to sparse domain

- If $t=1$

$$
\min _{\boldsymbol{\kappa}_{t}^{f}}\left\|\mathbf{K}_{t}^{f}-\boldsymbol{\Psi} \boldsymbol{\kappa}_{t}^{f}\right\|_{2}^{2} \quad \text { s.t. }\left\|\boldsymbol{\kappa}_{t}^{f}\right\|_{0} \leq p
$$

- else

$$
\boldsymbol{\kappa}_{t}^{f}=\boldsymbol{\kappa}_{t-1}^{a}
$$

3. Construct the new state vector

$$
\mathbf{X}_{t}^{f}=:\left[\boldsymbol{\kappa}_{t}^{f}, \boldsymbol{\Phi}_{t}^{f}, \mathbf{P}_{t}^{f}, \mathbf{S}_{t}^{f}\right]
$$

4. Perform EnKF analysis $\mathbf{y}_{t}$

$$
\mathbf{X}_{t}^{a}=\operatorname{EnKF}\left(\mathbf{X}_{t}^{f}, \mathbf{y}_{t}\right)
$$

where

$$
\mathbf{X}_{t}^{a}=:\left[\boldsymbol{\kappa}_{t}^{a}, \mathbf{\Phi}_{t}^{a}, \mathbf{P}_{t}^{a}, \mathbf{S}_{t}^{a}\right]
$$

5. Only retain the ' $p$ ' most significant weights for each ensemble member

$$
\max _{p+1: d}\left\{\boldsymbol{\kappa}_{t, i}^{a}\right\}=0 \quad \forall i=1: N e
$$

6. Transform back to spatial domain

$$
\mathbf{K}_{t}^{a}=\boldsymbol{\Psi} * \boldsymbol{\kappa}_{t}^{a}
$$

\section{Output: $\mathbf{K}_{t}^{a}$}

where $\mathbf{x}$ is the signal whose sparse solution is to be estimated and $\mathbf{y}$ is the measurement. The MMSE estimate by the SABMP is the sum over all 
possible $2^{n}$ supports $S$, found by searching the entire dimension $n$ of the signal $\mathbf{x}$. The SABMP algorithm has been shown to outperform most other standard algorithms in speed and computational efficiency, while providing similar or better estimation performances [27, 44].

The main input arguments to the algorithm for the problem under consideration are the observations $\mathbf{y}$, the dictionary $\boldsymbol{\Psi}$, and an initial estimate of the sparsity rate $p$. The SABMP algorithm also allows to refine the initial estimate of sparsity using an optional parameter $r_{\text {stop }}$, but this functionality is not used in our formulation. Information on the other optional algorithmic parameters and detailed derivations can be found in [27].

The Bayesian formulation of the SABMP enables fast and effective computation of solutions of a sparse problem [27]. In this paper; we compare the results of the OMP and SABMP algorithms and discuss the potential advantage of SABMP in improving the estimation accuracy in the context of the EnKF, while maintaining low computational complexity compared to OMP, especially at high sparsity rates.

\section{History Matching Setup}

\subsection{Reservoir Simulator}

The MATLAB Reservoir Simulation Toolbox (MRST) is a 2D finite difference two-phase flow reservoir simulator [45] coupling a well model to the two-phase flow problem for the oil and water phases governed by the system 
of equations [46]

$$
\begin{gathered}
\nabla \cdot v=q \\
v=-K\left[\lambda \nabla p+\left(\lambda_{w} \rho_{w}+\lambda_{o} \rho_{o}\right) g \nabla z\right],
\end{gathered}
$$

and

$$
\phi \frac{\partial s_{w}}{\partial t}+\nabla \cdot\left(f_{w}\left(s_{w}\right)\left[v+\lambda_{o}\left(\rho_{o}-\rho_{w}\right) g K \nabla z\right]\right)=q_{w}
$$

where $\lambda_{o}$ and $\lambda_{w}$ denote the individual mobilities, $\rho_{o}, \rho_{w}$ the densities, and $s_{o}, s_{w}$ the saturation, for the oil and water phases respectively, while their sums provide the same for the overall fluid. Furthermore, $q$ represents the flux, $f_{w}$ the fractional flow of the water phase, $\phi$ porosity of the formations, $v$ Darcy's velocity, $g$ the gravity, $K$ the permeability tensor, and $p$ the pressure within the reservoir. The solution to (6) and (7) is computed first for fluxes and pressure values while keeping fixed saturation. The computed fluxes and pressure values are then used to further estimate the saturation values using (8). The MRST simulator acts as the forward model to integrate the EnKF ensemble members forward in time.

Rock physics connect seismic data to the flow model and is described using a petro-elastic model (PEM). PEM is based on well log analysis, rock physics and available laboratory data and is composed of a set of equations used to calculate three elastic parameters, Poissons ratio, $\mathrm{P}$ impedance, and S impedance. The most commonly used equations are the Batzle and Wang equations for the fluid properties and the Gassmanns equations for rock properties [47], both adopted in this work. These relations predict elastic 
properties of saturated porous medium from simulated fluid and static rock properties, relating the fluid flow and wave propagation domains.

\subsection{Simulation Parameters}

We follow the same numerical setup as in [28, 29] and use a reservoir field stretching $0.5 \mathrm{~km}$ in both $\mathrm{x}$ and $\mathrm{y}$-directions and $10 \mathrm{~m}$ in the $\mathrm{z}$-direction. The reference permeability field is shown in Figure 1 where the flow channels having a high permeability value of 5000 millidarcy are easily distinguishable from the surrounding rock formations having much lower values of 100 millidarcy. The field is distributed on a grid of $50 \times 50 \times 1$ cells with the porosity values assumed constant at 30\%. Figure 1 also present the wells configuration, which possesses one injector well at the center of the domain and four producing wells at coordinate locations $(10,10),(10,40),(40,10)$ and $(40,40)$.

We first use a training image of a $239 \times 239$ dimension as shown in Figure 2 to generate a large set of 2000 realizations, each depicting a $50 \times 50$ size reservoir field with different subsurface geological structures using the S-GeMS software. S-GeMS implements the SNESIM (Single Normal Equation SIMulation) algorithm [48] for generating the realizations based on a probability model inferred from the training image. This enables these realizations to depict structures and connectivities similar to those in the training image. The S-GeMS realizations are used (i) to randomly select an initial ensemble for the EnKF, and (ii) as a training set for the K-SVD algorithm 
to generate a dictionary of 1000 linearly independent basis elements for the SGSD-EnKF algorithm. The complete SGSD-EnKF based history matching process is illustrated in Figure 2.

The MRST reservoir simulator was used to simulate flow, production, and seismic data based on the reference permeability shown in Figure 1. Observations are extracted from this reference run and include the Bottom hole pressure (BHP), water cut ratio (WCR), production flux and seismic measurements, each perturbed with independent noise, based on a diagonal observation error covariance matrix $\mathbf{R}_{k}$. The time-lapse seismic attributes used in the history matching setup are the Poisson's coefficients which give the ratio between the velocities of the primary seismic wave and the shear wave [49]. The Poisson's coefficients are highly sensitive to the fluid content in the geological formations due to the fact that the primary wave can propagate through the fluid, but not the shear wave. Consequently the Poisson's coefficients provide important information regarding the fluid flow in the medium and should help better reconstructing the channels structures through which the fluid flows, together with their boundaries and connectivities.

The standard deviation of observational errors is set to 290 psi for BHP, $25 \mathrm{~m}^{3} /$ day for the flux, around $7 \%$ for WCR and $15 \%$ for the seismic measurements. Production data are collected every 60 days while the seismic data are collected after every one year for a total history matching period of 5 years. The initial pressure level was set to 5076 psi for the injector 
well and 4350 psi for the producing wells. This history matching setup was used in the following numerical experiments for recovering the channel structures by estimating the permeability field with the standard EnKF and the SGSD-EnKF, using both the OMP and SABMP algorithms in the sparse transformation step.

To provide a quantitative analysis of recovering the channel structures and their locations, we use the Structural SIMilarity (SSIM) index from the image processing community [50]. The SSIM index is designed to measure the structural similarity in two images by performing a pixel-by-pixel comparison of the luminance, contrast and structural information; the last is measured in terms of the correlation between the two normalized images. We use SSIM here to compare the structural similarity of the estimated fields with the reference permeability field, where a high SSIM value indicates a good reconstruction quality. More information on the SSIM can be found in [50].

\section{Numerical Results}

We first present the results of the experiment when no seismic data is history matched for recovering the flow channel structures. Figure 4 presents the performance of the standard EnKF and the SGSD-EnKF, implemented with both the OMP and SABMP algorithm, using only production and flow data. An ensemble size of 80 members and a sparsity rate of $10 \%$ was used in this experiment to match the settings of [28, 29]. While the SGSD-EnKF

demonstrates a clear advantage over the standard EnKF in reconstructing 
the channels, it does not achieve as good contrast between high and low permeability values. The irregularities in channel reconstruction provide room for further improvement, which can potentially be addressed using seismic measurements.

Figure 5 presents the yearly estimates of the permeability fields over the 5 years history matching period using the standard EnKF and the SGSD-EnKF filter employing both OMP and SABMP algorithms, and history matching both production and time-lapse seismic data. The ensemble size is the same as in the previous experiment, while the inclusion of seismic data allows to increase the sparsity rate to $20 \%$. Suffering from the limitations discussed in Section 1, the standard EnKF struggles in recovering the subsurface flow channels even with the incorporation of seismic measurements. The performance of the SGSD-EnKF, particularly with the OMP algorithm, is much more robust, with the recovered fields exhibiting a close similarity with the reference permeability field. As for OMP, the SGSD-EnKF with SABMP also provides improved channels recovery over the standard EnKF. Time-lapse seismic data enhances the contrast between low and high permeability areas and further improves the channels reconstruction, enabling, for instance, the detection of the small rock feature in the top-left area of the reservoir, using both the OMP and SABMP algorithms. The quantitative analysis shown in terms of the SSIM index in Figure 6 also confirms the advantage of the SGSD-EnKF over the standard EnKF. The SGSD-EnKF with OMP provides significantly enhanced reconstruction after just two years of history 
matching, while its performance improves more gradually with the SABMP, outperforming the standard EnKF towards the end of the 5-years history matching period.

Figures 7 and 8 outline the performance of the EnKF and the SGSDEnKF against increasing ensemble sizes. The filter performances improve with increased ensemble sizes to a certain limit, as expected. Using more ensemble members than required results in degraded reconstruction for some of the larger ensemble sizes. The quantitative analysis of the reconstruction accuracy in Figure 9, in terms of SSIM, suggests that the SGSD-EnKF, particularly when used with the OMP algorithm, continues to provide a clear advantage over the EnKF for the given range of ensemble sizes. While the performance of the SGSD-EnKF with the SABMP algorithm was only marginally better than the standard EnKF with an ensemble of 80 members, the improvement is much more pronounced for most larger ensemble sizes. It is also interesting to note that the reconstruction quality with the SGSDEnKF scheme using the OMP algorithm and an ensemble of 80 members is almost as good as that estimated by the standard EnKF using twice as many members. This demonstrates the important advantage of incorporating prior information through the structurally diverse basis elements in the SGSDEnKF scheme.

An analysis of the computational complexity is presented in Figure 10 for all three filters, outlining the increase in the overall simulation time due to the one-time sparse transformation step in the SGSD-EnKF scheme, against 
the standard EnKF. The figure clearly depicts the linear increase in computational time expected from the increased ensembles and highlights the limitation arising from the added computational burden when using large ensembles.

In Figure 11, we conduct a sensitivity analysis for the SGSD-EnKF scheme using both the OMP and SABMP algorithms with a fixed ensemble size of 80 members and sparsity rates varying between 10 - 50\%, consistent with the analysis in [29]. As seen in previous experiments, SGSD-EnKF performs significantly better than the standard EnKF regardless of the choice of the OMP or the Bayesian SABMP algorithm for enforcing sparsity. The quantitative analysis in terms of the SSIM index in Figure 12 confirms this behavior, with the OMP algorithm resulting in major improvement in reconstruction quality with a sparsity rate of just $20 \%$. The sudden drop in SSIM value at $40 \%$ sparsity rate for OMP can be explained by the fact that the OMP algorithm is being forced to use too many basis element to represent the subsurface field and the particular combination of basis element results in significantly degraded performance. The rise again in SSIM value at $50 \%$ sparsity rate is a result of some of these negative effects being balanced out by using even more basis elements. The performance with the SABMP algorithm improves more gradually over the given range of sparsity rates, achieving comparable SSIM values at $50 \%$ sparsity. Due to differences in their formulation, OMP and SABMP algorithms achieve their best reconstruction at different sparsity rates. A comparison of the computational times, however, reveals a signifi- 
cant drawback of OMP over SABMP. Figure 13 plots the computational time taken by the SGSD-EnKF algorithm when implemented with both OMP and SABMP against given sparsity rates. While OMP provides better estimates, its computational complexity increases linearly with increasing sparsity rates. This trend imposes a severe limitation on using OMP when more basis elements need to be included for enhanced channels reconstruction to exploit the additional information available from the seismic measurements. The increase in computational cost for the SABMP algorithm is minimal compared to OMP. The Bayesian formulation of the SABMP helps achieving enhanced reconstruction with the SGSD-EnKF (compared to the standard EnKF), as measured by improved SSIM values, while adding lower computational costs compared to what would be incurred from using the OMP with SGSD-EnKF. This suggests SABMP to be a viable alternative to OMP, where a minor trade-off against the reconstruction quality might be deemed acceptable to reduce the computational requirements.

\section{Summary and Discussion}

We investigated the effects of incorporating time-lapse seismic measurements on the channel reconstruction performance with the SGSD-EnKF algorithm under a new sparse Bayesian framework. As previously reported in [28] and [29], transformation to the sparse geological domain generated by training over large collection of geological structures helps incorporating useful prior information in the EnKF estimation process. This leads to enhanced 
estimation of the subsurface permeability field, better recovering the geological channels structures, their thicknesses and connectivities. The inclusion of seismic data greatly helps in further improving the reconstruction of the flow channels, with the SGSD-EnKF demonstrating a clear advantage over the standard EnKF. The Bayesian SABMP sparse reconstruction algorithm emerges as a good alternative to the OMP algorithm proposed in the original SGSD-EnKF scheme, with a computational complexity that scales much slower compared to the OMP algorithm against increasing sparsity rates. It enables effective use of more basis elements for enhanced reconstruction with the seismic measurements. The choice of SABMP over OMP is dependent on the acceptable level of trade-off between the computational complexity and reconstruction quality. The SGSD-EnKF performs better than the standard EnKF in all cases, regardless of the choice of the sparse reconstruction algorithm.

\section{Acknowledgement}

This work was funded in part by a CRG2 grant CRG \_R2 \_13\_ALOU \_KAUST \_2 from the Office of Competitive Research (OCRF) at King Abdullah University of Science and Technology (KAUST), Thuwal, Saudi Arabia. 


\section{References}

[1] D. S. Oliver and Y. Chen, "Recent progress on reservoir history matching: a review," Computational Geosciences, vol. 15, no. 1, pp. 185-221, 2011.

[2] M. J. Ronayne, S. M. Gorelick, and J. Caers, "Identifying discrete geologic structures that produce anomalous hydraulic response: An inverse modeling approach," Water Resources Research, vol. 44, no. 8, p. W08426, 2008.

[3] A. Elsheikh, M. Wheeler, and I. Hoteit, "Sparse calibration of subsurface flow models using nonlinear orthogonal matching pursuit and an iterative stochastic ensemble method," Advances in Water Resources, vol. 56, no. 0, pp. 14-26, 2013.

[4] B. Jafarpour, D. B. McLaughlin, and Others, "Estimating channelizedreservoir permeabilities with the ensemble Kalman filter: The importance of ensemble design," Spe Journal, vol. 14, no. 02, pp. 374-388, 2009.

[5] B. Jafarpour and M. Khodabakhshi, "A probability conditioning method (PCM) for nonlinear flow data integration into multipoint statistical facies simulation," Mathematical Geosciences, vol. 43, no. 2, pp. 133$164,2011$.

[6] M. Khodabakhshi and B. Jafarpour, "A Bayesian mixture-modeling approach for flow-conditioned multiple-point statistical facies simulation from uncertain training images," Water Resources Research, 2013.

[7] G. Evensen, "The Ensemble Kalman Filter: Theoretical formulation and practical implementation," Ocean Dynamics, vol. 53, no. 4, pp. 343-367, nov 2003. [Online]. Available: http://link.springer.com/10.1007/s10236003-0036-9

[8] S. Aanonsen, D. Oliver, A. Reynolds, and B. Vallis, "The Ensemble Kalman Filter in Reservoir Engineering-a Review," SPE Journal, vol. 14, no. 3, pp. 393-412, 2009. 
[9] O. M. Bucci, L. Crocco, T. Isernia, and V. Pascazio, "Subsurface inverse scattering problems: quantifying, qualifying, and achieving the available information," Geoscience and Remote Sensing, IEEE Transactions on, vol. 39, no. 11, pp. 2527-2538, nov 2001.

[10] J. Mandel, Efficient implementation of the ensemble Kalman filter. University of Colorado at Denver and Health Sciences Center, Center for Computational Mathematics, 2006.

[11] B. Jafarpour, V. K. Goyal, D. B. McLaughlin, and W. T. Freeman, "Compressed history matching: exploiting transform-domain sparsity for regularization of nonlinear dynamic data integration problems," Mathematical Geosciences, vol. 42, no. 1, pp. 1-27, 2010.

[12] — , "Transform-domain sparsity regularization for inverse problems in geosciences," Geophysics, vol. 74, no. 5, pp. R69—R83, 2009.

[13] E. J. Candes, Y. C. Eldar, D. Needell, and P. Randall, "Compressed sensing with coherent and redundant dictionaries," Applied and Computational Harmonic Analysis, vol. 31, no. 1, pp. 59-73, 2011.

[14] D. L. Donoho, "Compressed sensing," Information Theory, IEEE Transactions on, vol. 52, no. 4, pp. 1289-1306, apr 2006.

[15] L. Li and B. Jafarpour, "Effective solution of nonlinear subsurface flow inverse problems in sparse bases," Inverse Problems, vol. 26, p. 105016, 2010 .

[16] M.-r. Khaninezhad, B. Jafarpour, and S. California, "Sparse Geologic Dictionaries for Field-Scale History Matching Application," no. February, pp. 23-25, 2015.

[17] M. M. Khaninezhad, B. Jafarpour, and L. Li, "Sparse geologic dictionaries for subsurface flow model calibration: Part I. Inversion formulation," Advances in Water Resources, vol. 39, pp. 106-121, 2012.

[18] M. Khaninezhad, B. Jafarpour, and M. M. Khaninezhad, "Hybrid Parameterization for Robust History Matching," SPE Journal, no. June, 2013. [Online]. Available: https://www.onepetro.org/journalpaper/SPE-146934-PA 
[19] B. Jafarpour and D. B. McLaughlin, "History matching with an ensemble Kalman filter and discrete cosine parameterization," Computational Geosciences, vol. 12, no. 2, pp. 227-244, 2008.

[20] B. Jafarpour, "Wavelet reconstruction of geologic facies from nonlinear dynamic flow measurements," Geoscience and Remote Sensing, IEEE Transactions on, vol. 49, no. 5, pp. 1520-1535, 2011.

[21] L. Li and B. Jafarpour, "A sparse Bayesian framework for conditioning uncertain geologic models to nonlinear flow measurements," Advances in Water Resources, vol. 33, no. 9, pp. 1024-1042, 2010.

[22] J. Lee and P. K. Kitanidis, "Bayesian inversion with total variation prior for discrete geologic structure identification," Water Resources Research, vol. 49, no. 11, pp. 7658-7669, 2013.

[23] M. M. Khaninezhad, B. Jafarpour, and L. Li, "Sparse geologic dictionaries for subsurface flow model calibration: Part II. Robustness to uncertainty," Advances in Water Resources, vol. 39, pp. 106-121, 2012.

[24] I. Hoteit and A. Köhl, "Efficiency of reduced-order, time-dependent adjoint data assimilation approaches," Journal of oceanography, vol. 62, no. 4, pp. 539-550, 2006.

[25] M. U. Altaf, M. Ambrozic, M. F. McCabe, and I. Hoteit, "A study of reduced-order 4DVAR with a finite element shallow water model," International Journal for Numerical Methods in Fluids, 2015.

[26] J. A. Tropp and A. C. Gilbert, "Signal recovery from random measurements via orthogonal matching pursuit," Information Theory, IEEE Transactions on, vol. 53, no. 12, pp. 4655-4666, 2007.

[27] M. Masood and T. Al-Naffouri, "Sparse reconstruction using distribution agnostic Bayesian matching pursuit," Signal Processing, IEEE Transactions on, vol. 61, no. 21, pp. 5398-5309, 2013.

[28] F. Sana, K. Katterbauer, T. Al-Naffouri, and I. Hoteit, "Enhanced Recovery of Subsurface Geological Structures Using Compressed Sensing And The Ensemble Kalman Filter," in International Geoscience and Remote Sensing Symposium 2015 (IGARSS 2015), 2015. 
[29] — - "Orthogonal Matching Pursuit for Enhanced Recovery of Sparse Geological Structures with the Ensemble Kalman Filter," IEEE Journal of Selected Topics in Applied Earth Observations and Remote Sensing (JSTARS), 2016.

[30] D. E. Lumley, R. A. Behrens, and Others, "Practical issues of 4D seismic reservoir monitoring: What an engineer needs to know," SPE Reservoir Evaluation \& Engineering, vol. 1, no. 06, pp. 528-538, 1998.

[31] K. Aid and P. G. Richards, "Quantitative seismology: Theory and methods," San Francisco, 1980.

[32] D.-h. Han and M. L. Batzle, "Gassmann's equation and fluid-saturation effects on seismic velocities," Geophysics, vol. 69, no. 2, pp. 398-405, 2004.

[33] T. Oldenziel, Time-lapse seismic within reservoir engineering. TU Delft, Delft University of Technology, 2003.

[34] J.-A. Skjervheim, G. Evensen, S. I. Aanonsen, B. O. Ruud, T.-A. Johansen, and Others, "Incorporating 4D seismic data in reservoir simulation models using ensemble Kalman filter," in SPE Annual Technical Conference and Exhibition. Society of Petroleum Engineers, 2005.

[35] O. Gosselin, S. I. Aanonsen, I. Aavatsmark, A. Cominelli, R. Gonard, M. Kolasinski, F. Ferdinandi, L. Kovacic, K. Neylon, and Others, "History matching using time-lapse seismic (HUTS)," in SPE Annual Technical Conference and Exhibition. Society of Petroleum Engineers, 2003.

[36] K. Katterbauer and I. Hoteit, "Improving Reservoir History Matching of EM Heated Heavy Oil Reservoirs via Cross-well Seismic Tomography," in SPE Heavy Oil Conference-Canada. Society of Petroleum Engineers, 2014.

[37] K. Katterbauer, I. Hoteit, and S. Sun, "EMSE: Synergizing EM and seismic data attributes for enhanced forecasts of reservoirs," Journal of Petroleum Science and Engineering, 2014.

[38] F. Ravanelli, I. Hoteit, and Others, "Using Crosswell Data To Enhance History Matching," in SPE Bergen One Day Seminar. Society of Petroleum Engineers, 2014. 
[39] O. Leeuwenburgh, J. Brouwer, and M. Trani, "Ensemble-based conditioning of reservoir models to seismic data," Computational Geosciences, vol. 15, no. 2, pp. 359-378, 2011.

[40] K. Katterbauer, S. Arango, S. Sun, and I. Hoteit, "Multidata reservoir history matching for enhanced reservoir forecasting and uncertainty quantification," Journal of Petroleum Science and Engineering, vol. 128, no. 0, pp. 160-176, 2015. [Online]. Available: http://www.sciencedirect.com/science/article/pii/S0920410515000686

[41] I. Hoteit, X. Luo, and D.-T. Pham, "Particle Kalman Filtering: A Nonlinear Bayesian Framework for Ensemble Kalman Filters," Monthly Weather Review, vol. 140, pp. 528-542, 2012.

[42] X. Luo, I. Hoteit, L. Duan, and W. Wang, "Review of nonlinear Kalman, ensemble and particle filtering with application to the reservoir history matching problem," Nonlinear Estimation and Applications to Industrial Systems Control, 2011.

[43] M. Aharon, M. Elad, and A. Bruckstein, "K-SVD: An Algorithm for Designing Overcomplete Dictionaries for Sparse Representation," IEEE Transactions on Signal Processing, vol. 54, no. 11, pp. 4311-4322, 2006.

[44] F. Sana, T. Ballal, T. Y. Al-Naffouri, and I. Hoteit, "Low-complexity wireless monitoring of respiratory movements using ultra-wideband impulse response estimation," Biomedical Signal Processing and Control, vol. 10, pp. 192-200, 2014.

[45] K. Lie, S. Krogstad, I. Ligaarden, and Others, "Open-source MATLAB implementation of consistent discretisations on complex grids," Computational Geosciences, vol. 16, no. 2, pp. 297-322, 2012.

[46] Z. Chen, Reservoir Simulation: Mathematical Techniques in Oil Recovery, ser. CBMS-NSF regional conference series in applied mathematics. Society for Industrial and Applied Mathematics (SIAM), 2007.

[47] E. Reiso, M. C. Haver, M. Aga, and Others, "Integrated workflow for quantitative use of time-lapse seismic data in history matching: A North Sea field case," in SPE Europec/EAGE Annual Conference. Society of Petroleum Engineers, 2005. 
[48] S. Strebelle, "Conditional simulation of complex geological structures using multiple-point statistics," Mathematical Geology, vol. 34, no. 1, pp. 1-21, 2002.

[49] N. I. Christensen, "Poisson's ratio and crustal seismology," Journal of Geophysical Research: Solid Earth (1978-2012), vol. 101, no. B2, pp. 3139-3156, 1996.

[50] Z. Wang, A. C. Bovik, H. R. Sheikh, and E. P. Simoncelli, "Image quality assessment: from error visibility to structural similarity," Image Processing, IEEE Transactions on, vol. 13, no. 4, pp. 600-612, 2004. 


\section{List of Figures}

1 (a): Reference permeability field composed of high permeability areas plotted in red indicating flow channels and low permeability areas plotted in blue indicating surrounding rock formations. (b): Injector-producer pattern showing the distribution of the injector wells (black circles) and producer wells (white circles) on top of the reference permeability field. . . . 36

2 The sparse history matching framework with the SGSD-EnKF algorithm. The training image in panel (a) is used to generate multiple realizations of subsurface geological structures (examples of these are shown in panel (b)) using the SNESIM algorithm. These realizations are later used as input to the K-SVD algorithm to generate a dictionary of linearly independent basis elements as outlined in panel (c). A weighted sparse linear combination of these basis elements can be used to represent the ensemble members in the sparse dictionary domain as schematized in panel (d). The EnKF update step on the ensemble members is carried out in the sparse dictionary domain as shown in panel (e). Finally, the ensemble members are transformed back to spatial domain to give the recovered permeability field as depicted in (f) . . . . . . . . 37

3 Illustration of the Ensemble Kalman Filter based history match-

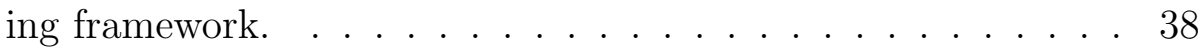


4 1st Row: Reference Permeability field, Rows 2 to 6: Recovered fields after 1, 2, 3, 4 and 5 years of history matching respectively with the Standard EnKF (left column), SGSDEnKF with OMP (middle column), and SGSD-EnKF with SABMP (right column) for the case when no seismic data is history matched for recovering the flow channels structures. All values and scales are in units of millidarcy. The filters are implemented with an ensemble of 80 members and 10\% basis elements from the K-SVD dictionary for the SGSD-EnKF. . . 39

5 1st Row: Reference Permeability field, Rows 2 to 6: Recovered fields after 1, 2, 3, 4 and 5 years of history matching respectively with the Standard EnKF (left column), SGSD-EnKF with OMP (middle column), and SGSD-EnKF with SABMP (right column) for the case when both flow and seismic data are history matched for recovering the flow channels structures. All values and scales are in units of millidarcy. The filters are implemented with an ensemble of 80 members and $20 \%$ basis elements from the K-SVD dictionary for the SGSDEnKF. .................... . . 40 
6 Estimation quality of the recovered permeability field, in terms of the SSIM, over the 5-years history matching period for the experiment shown in Figure 5, where a high value of SSIM indicates that the estimated field resembles more closely with the reference permeability field shown in Figure 1. . . . . . . 41

7 1st Row: Reference Permeability field, Rows 2 to 6: Recovered fields at the end of 5-years history matching period with an ensemble size of 40, 80, 120, 160 and 200 respectively with the Standard EnKF (left column), SGSD-EnKF with OMP (middle column), and SGSD-EnKF with SABMP (right column). All values and scales are in units of millidarcy. The filters are implemented using $20 \%$ basis elements from the K-SVD dictionary for the SGSD-EnKF. . . . . . . . . . . . . . 42

8 1st Row: Reference Permeability field, Rows 2 to 6: Recovered fields at the end of 5-years history matching period with an ensemble size of 240, 280, 320, 360 and 400 respectively with the Standard EnKF (left column), SGSD-EnKF with OMP (middle column), and SGSD-EnKF with SABMP (right column). All values and scales are in units of millidarcy. The filters are implemented using $20 \%$ basis elements from the K-SVD dictionary for the SGSD-EnKF. . . . . . . . . . . . . . . . . 43 
9 Estimation quality of the recovered permeability field, in terms of the SSIM, against increasing ensembles sizes for the experiment shown in Figure 7 and Figure 8. . . . . . . . . . . . . . 44

10 Analysis of computational times against increasing ensembles sizes for the experiment shown in Figure 7 and Figure 8. . . . 45

11 1st Row: Reference Permeability field, Rows 2 to 6: Recovered fields at the end of 5-years history matching period with sparsity rates of $10 \%, 20 \%, 30 \%, 40 \%$ and $50 \%$ respectively using SGSD-EnKF with OMP (left column), and SGSD-EnKF with SABMP (right column). All values and scales are in units of millidarcy. All filters are implemented with an ensemble of 80 members. ................... . . . 46

12 Estimation quality of the recovered permeability field, in terms of the SSIM, against increasing sparsity rates for the experiment shown in Figure 11. . . . . . . . . . . . . . . . . 47

13 Analysis of computational times against increasing sparsity rates for the experiment shown in Figure 11. . . . . . . . . . . 48 
(a) Reference Permeability Field

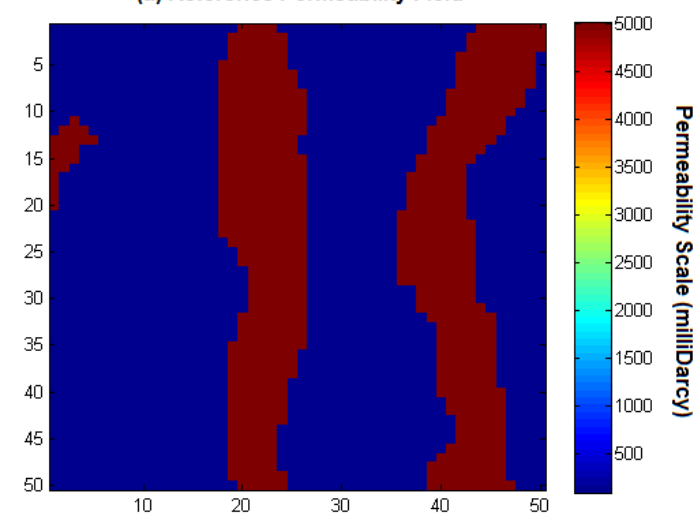

(b) Injector-Producer Pattern

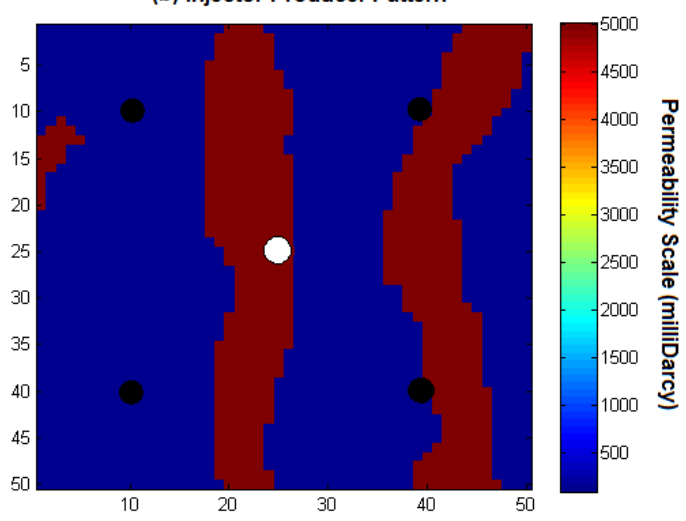

Figure 1: (a): Reference permeability field composed of high permeability areas plotted in red indicating flow channels and low permeability areas plotted in blue indicating surrounding rock formations. (b): Injector-producer pattern showing the distribution of the injector wells (black circles) and producer wells (white circles) on top of the reference permeability field. 
(b) Realizations of Complex

Geological Structures

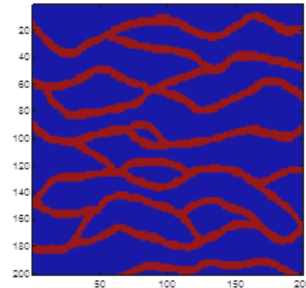

(a) Training Image
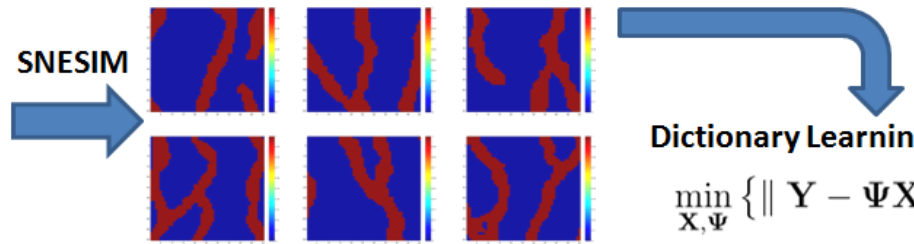

Dictionary Learning: K-SVD

$$
\min _{\mathbf{X}, \Psi}\left\{\|\mathbf{Y}-\Psi \mathrm{X}\|_{2}^{2}\right\}
$$
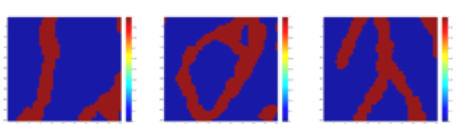

s.t. $\left\|\mathbf{x}_{i}\right\|_{0} \leq \mathbf{T}_{0} \forall i=1, \ldots, N_{t}$
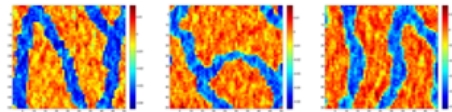

(d) Dictionary Weights for Permeability in Sparse Domain
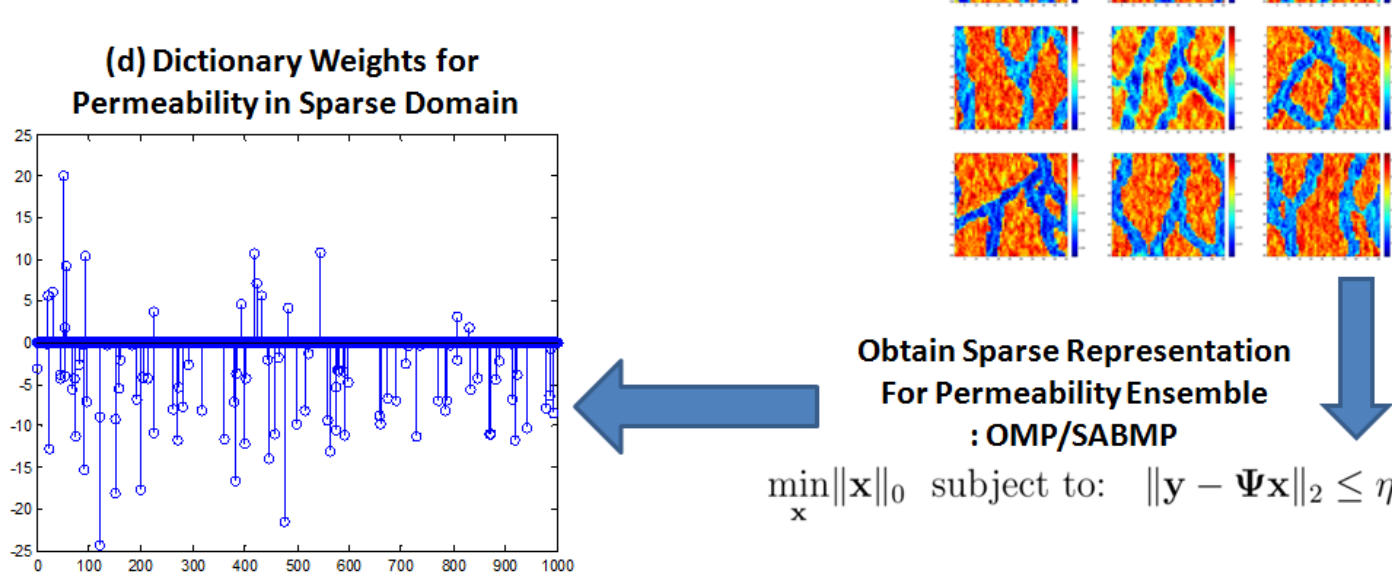

듬
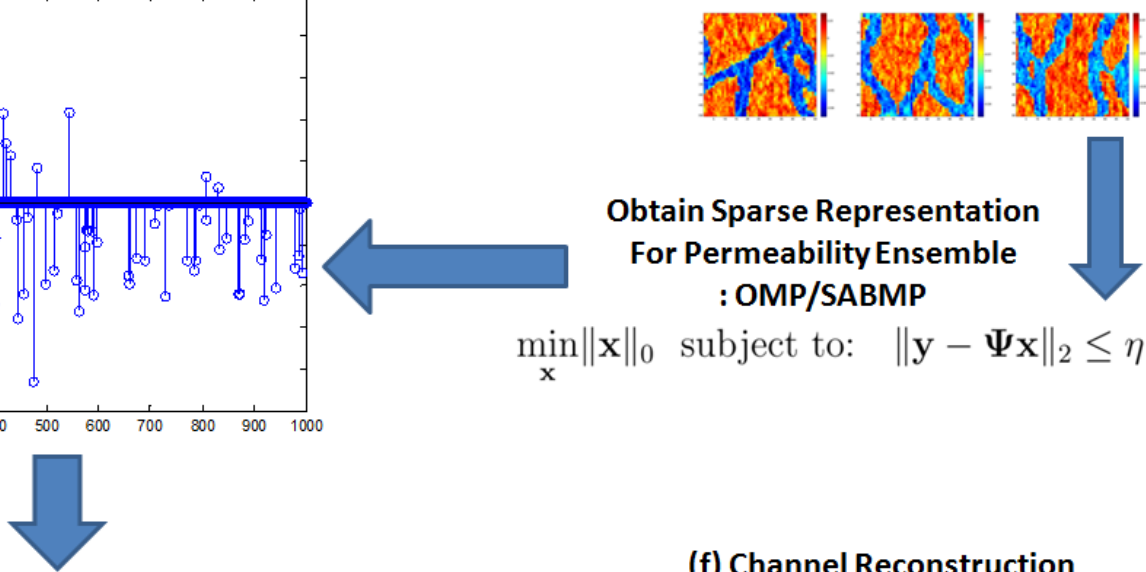

(e) History Matching with the EnKF

(f) Channel Reconstruction in Spatial Domain
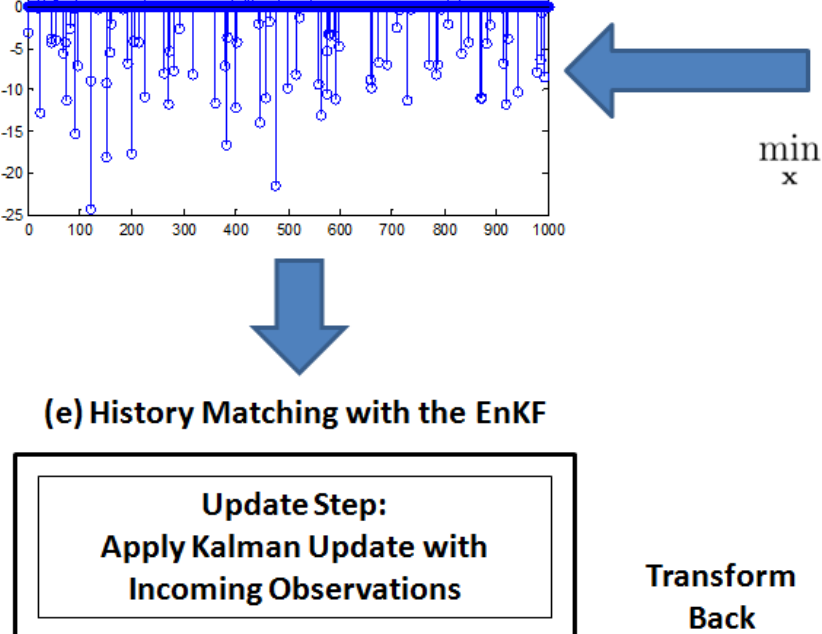

Obtain Sparse Representation

For Permeability Ensemble : OMP/SABMP

$\min _{\mathbf{x}}\|\mathbf{x}\|_{0}$ subject to: $\left\|\mathbf{y}-\Psi_{\mathbf{x}}\right\|_{2} \leq \eta$,
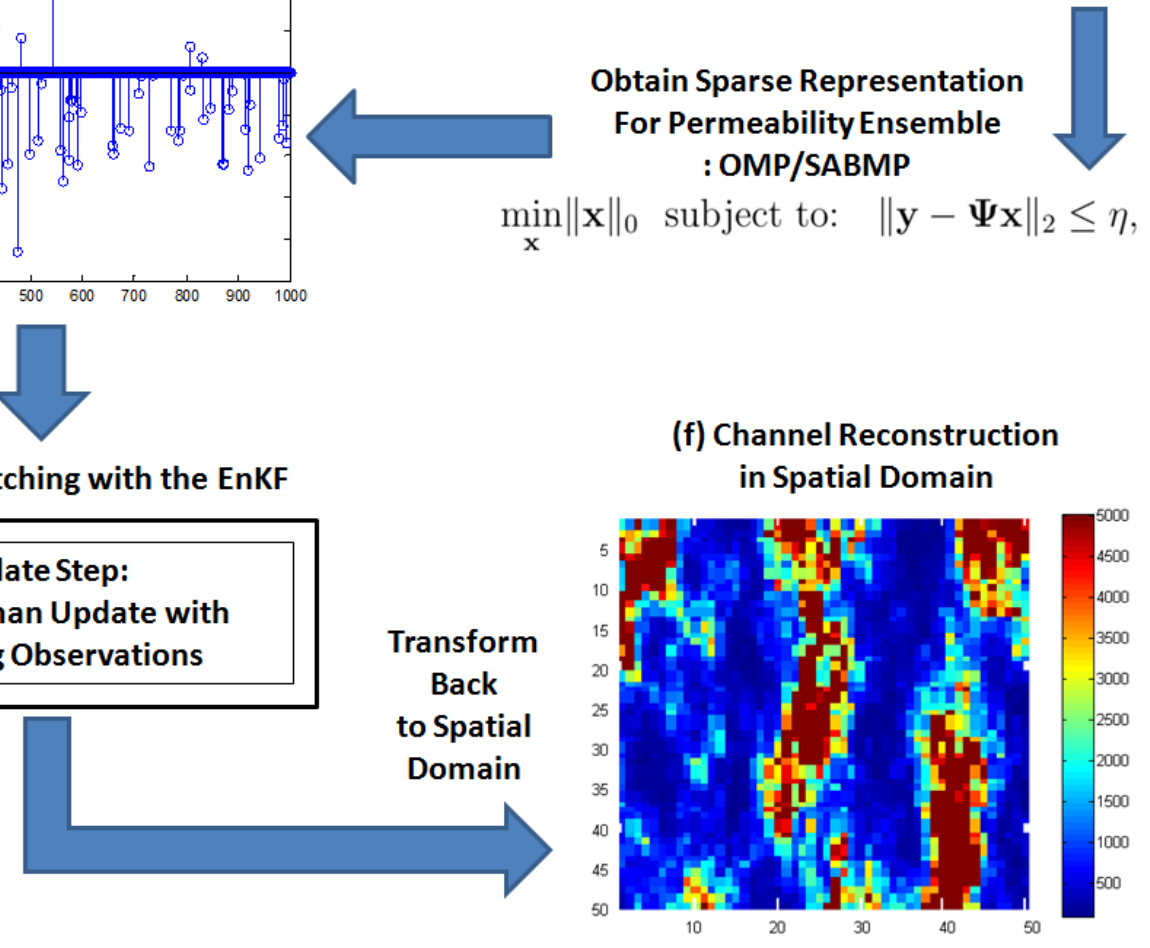

Figure 2: The sparse history matching framework with the SGSD-EnKF algorithm. The training image in panel (a) is used to generate multiple realizations of subsurface geological structures (examples of these are shown in panel (b)) using the SNESIM algorithm. These realizations are later used as input to the K-SVD algorithm to generate a dictionary of linearly independent basis elements as outlined in panel (c). A weighted sparse linear combination of these basis elements can be used to represent the ensemble members in the sparse dictionary domain as schematized in panel (d). The EnKF update step on the ensemble members is carried out in the sparse dictionary domain as shown in panel (e). Finally, the ensemble members are transformed back to spatial domain to give the recovered permeability field as depicted in (f). 

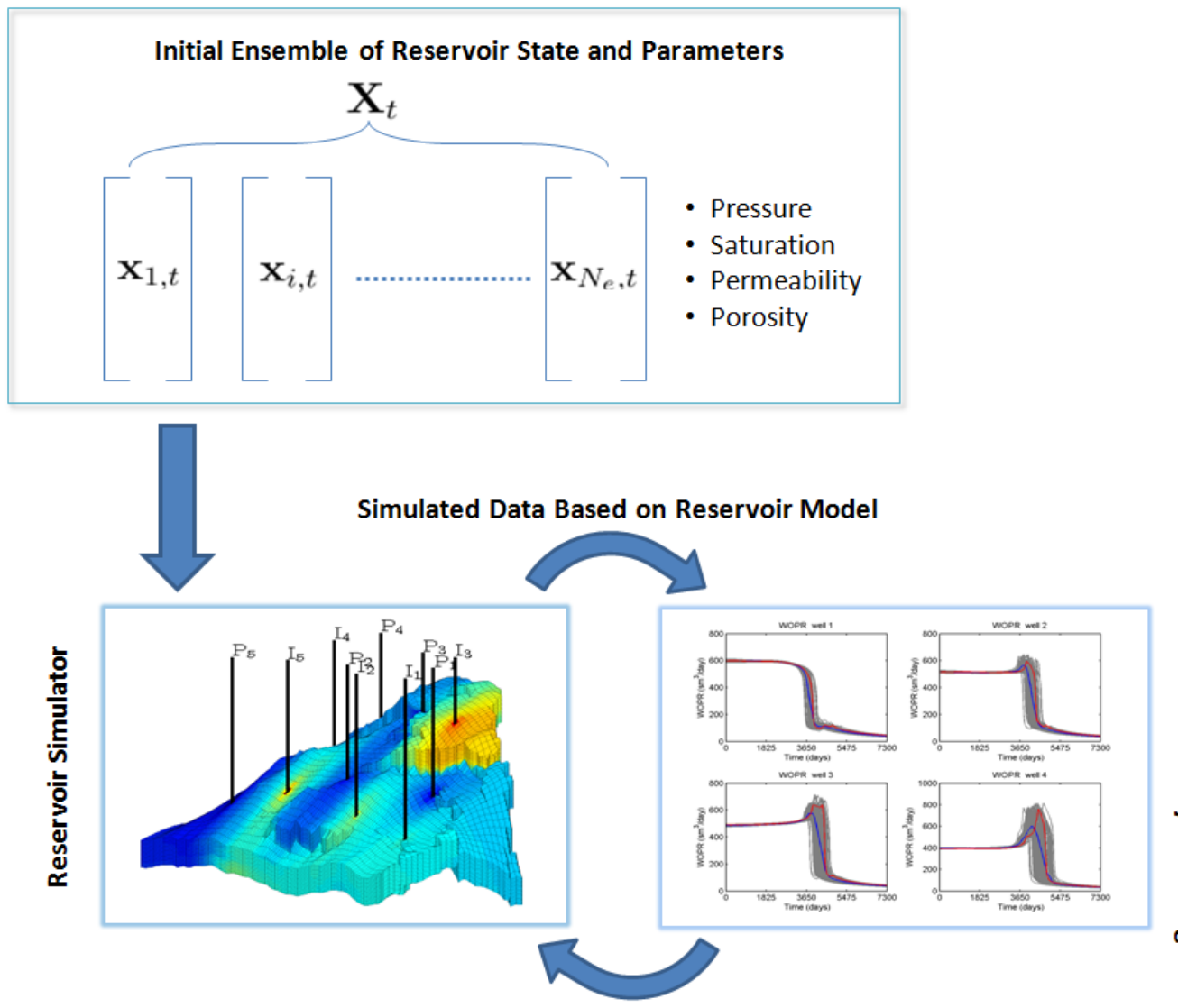

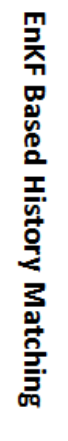

Calibrated Reservoir Parameters Based on Observed Data

Figure 3: Illustration of the Ensemble Kalman Filter based history matching framework. 


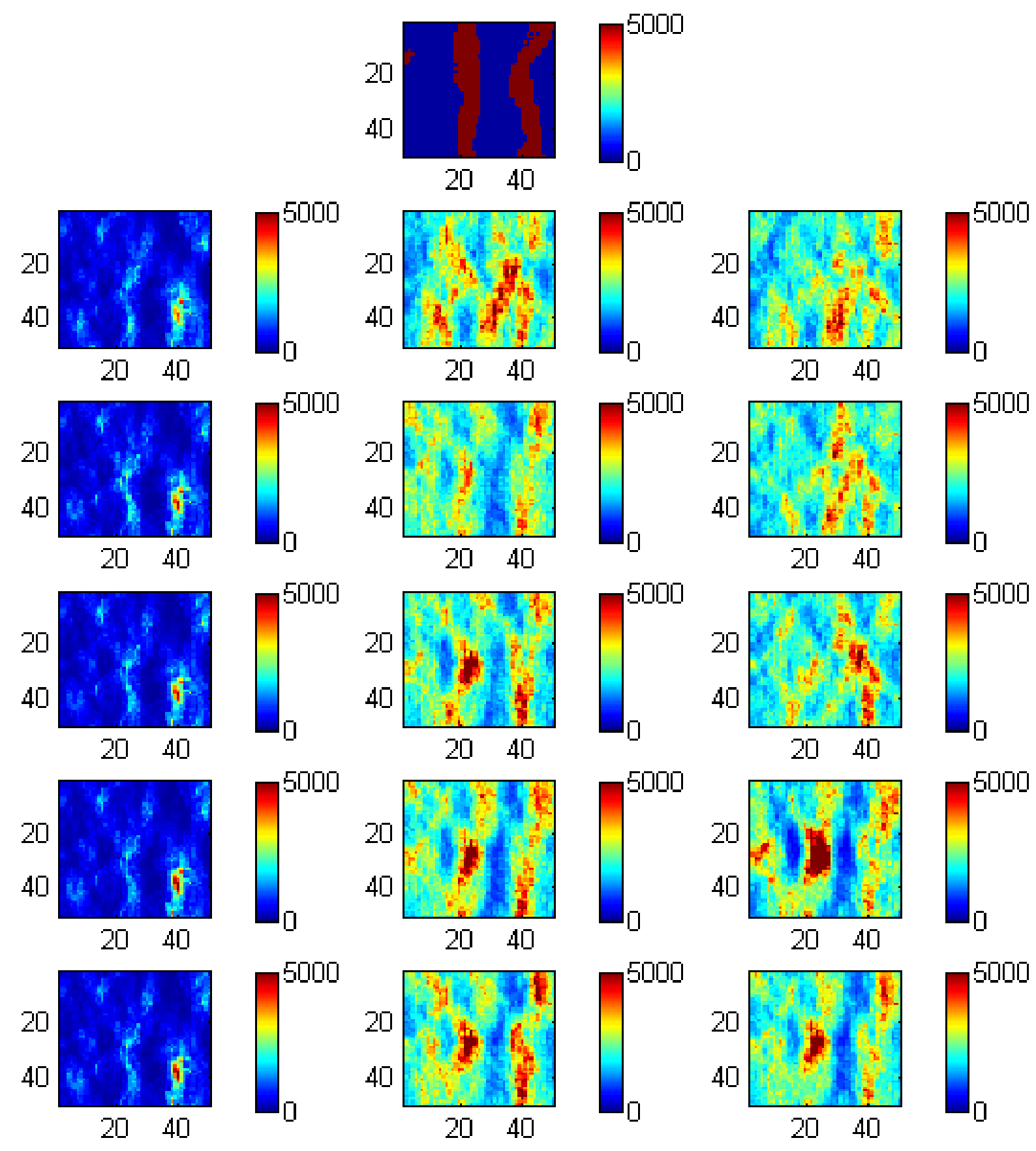

Figure 4: 1st Row: Reference Permeability field, Rows 2 to 6: Recovered fields after 1, 2, 3,4 and 5 years of history matching respectively with the Standard EnKF (left column), SGSD-EnKF with OMP (middle column), and SGSD-EnKF with SABMP (right column) for the case when no seismic data is history matched for recovering the flow channels structures. All values and scales are in units of millidarcy. The filters are implemented with an ensemble of 80 members and $10 \%$ basis elements from the K-SVD dictionary for the SGSD-EnKF. 


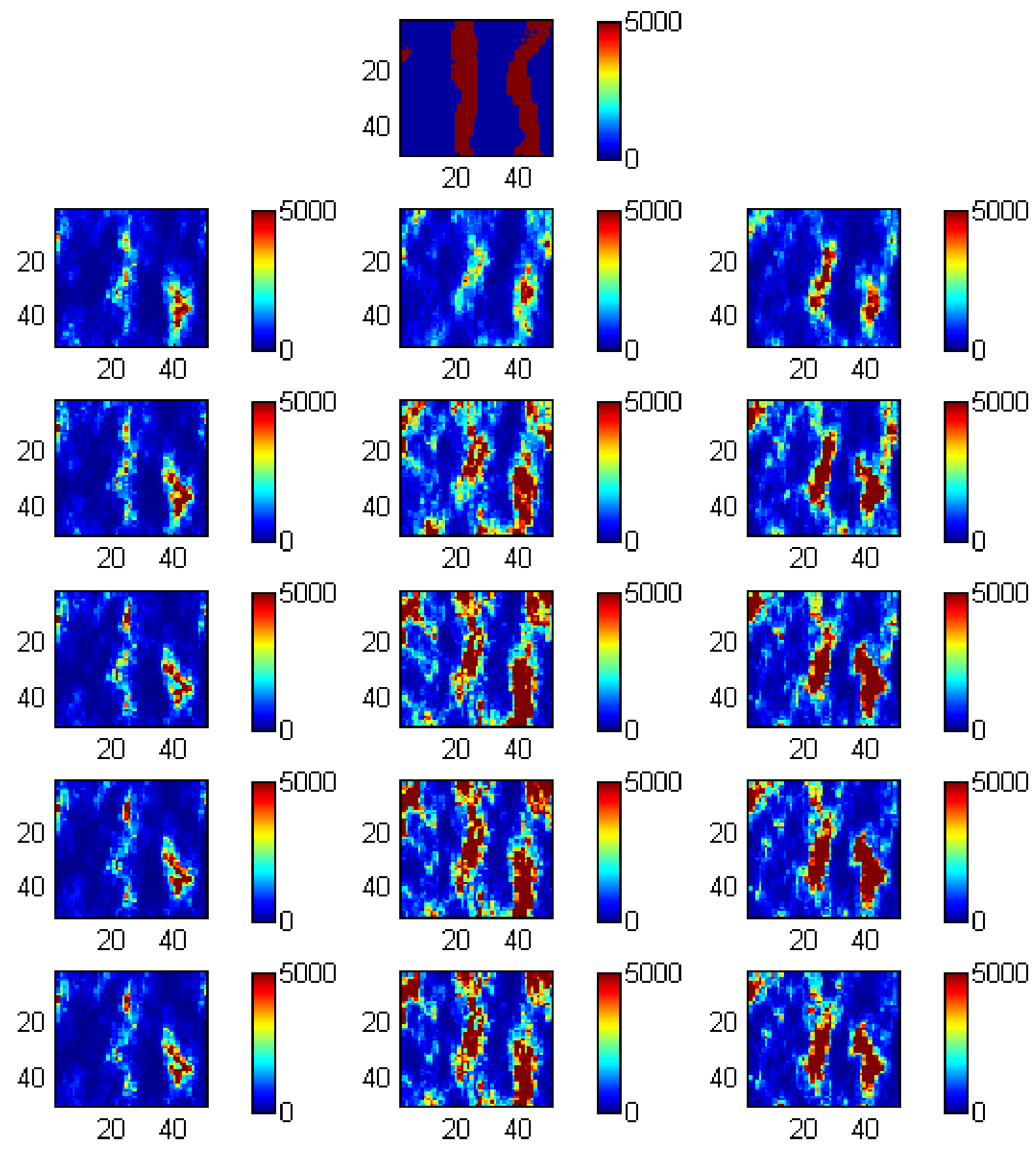

Figure 5: 1st Row: Reference Permeability field, Rows 2 to 6: Recovered fields after 1, 2, 3, 4 and 5 years of history matching respectively with the Standard EnKF (left column), SGSD-EnKF with OMP (middle column), and SGSD-EnKF with SABMP (right column) for the case when both flow and seismic data are history matched for recovering the flow channels structures. All values and $\$$ gales $^{2}$ are in units of millidarcy. The filters are implemented with an ensemble of 80 members and $20 \%$ basis elements from the K-SVD dictionary for the SGSD-EnKF. 


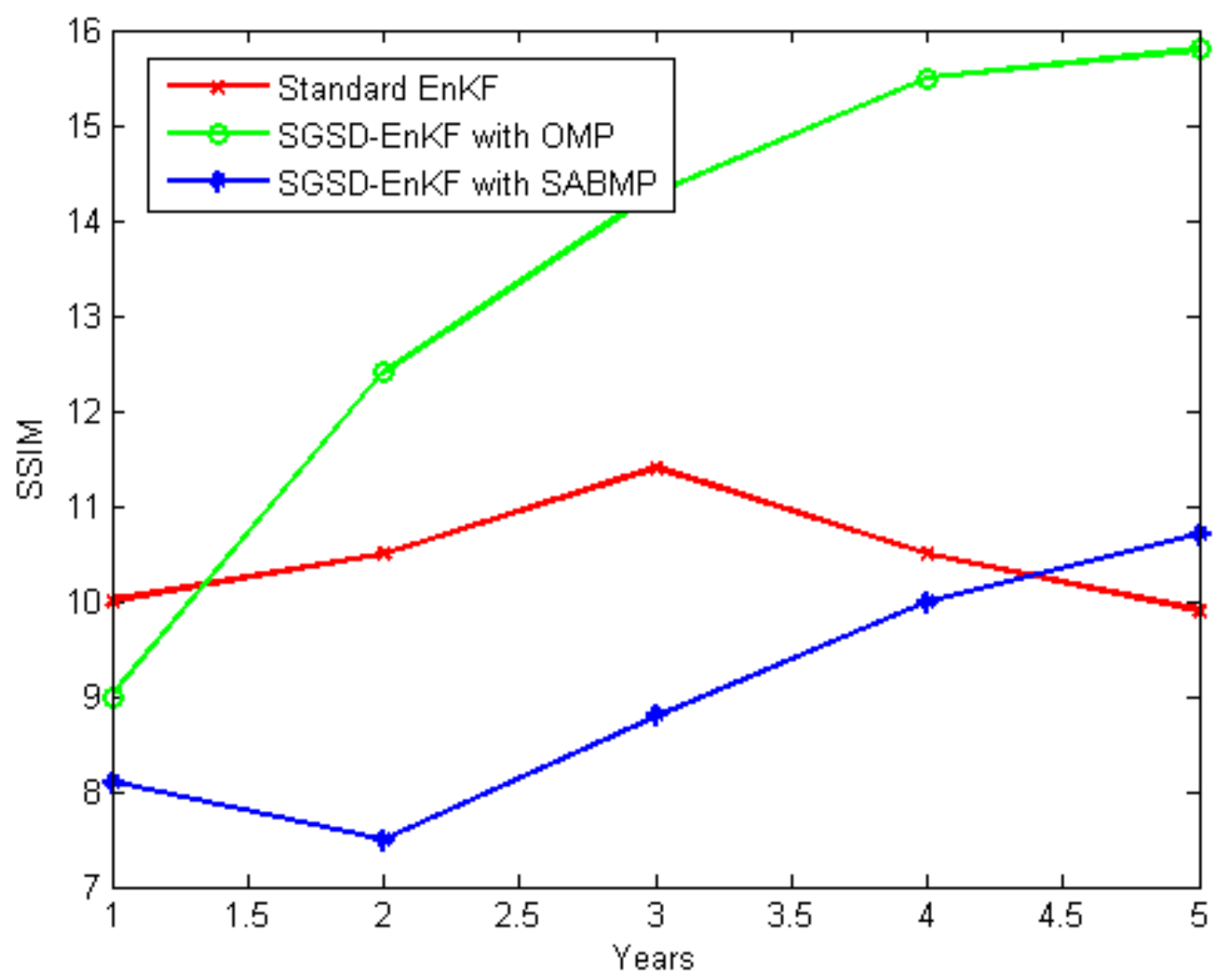

Figure 6: Estimation quality of the recovered permeability field, in terms of the SSIM, over the 5-years history matching period for the experiment shown in Figure 5, where a high value of SSIM indicates that the estimated field resembles more closely with the reference permeability field shown in Figure 1. 


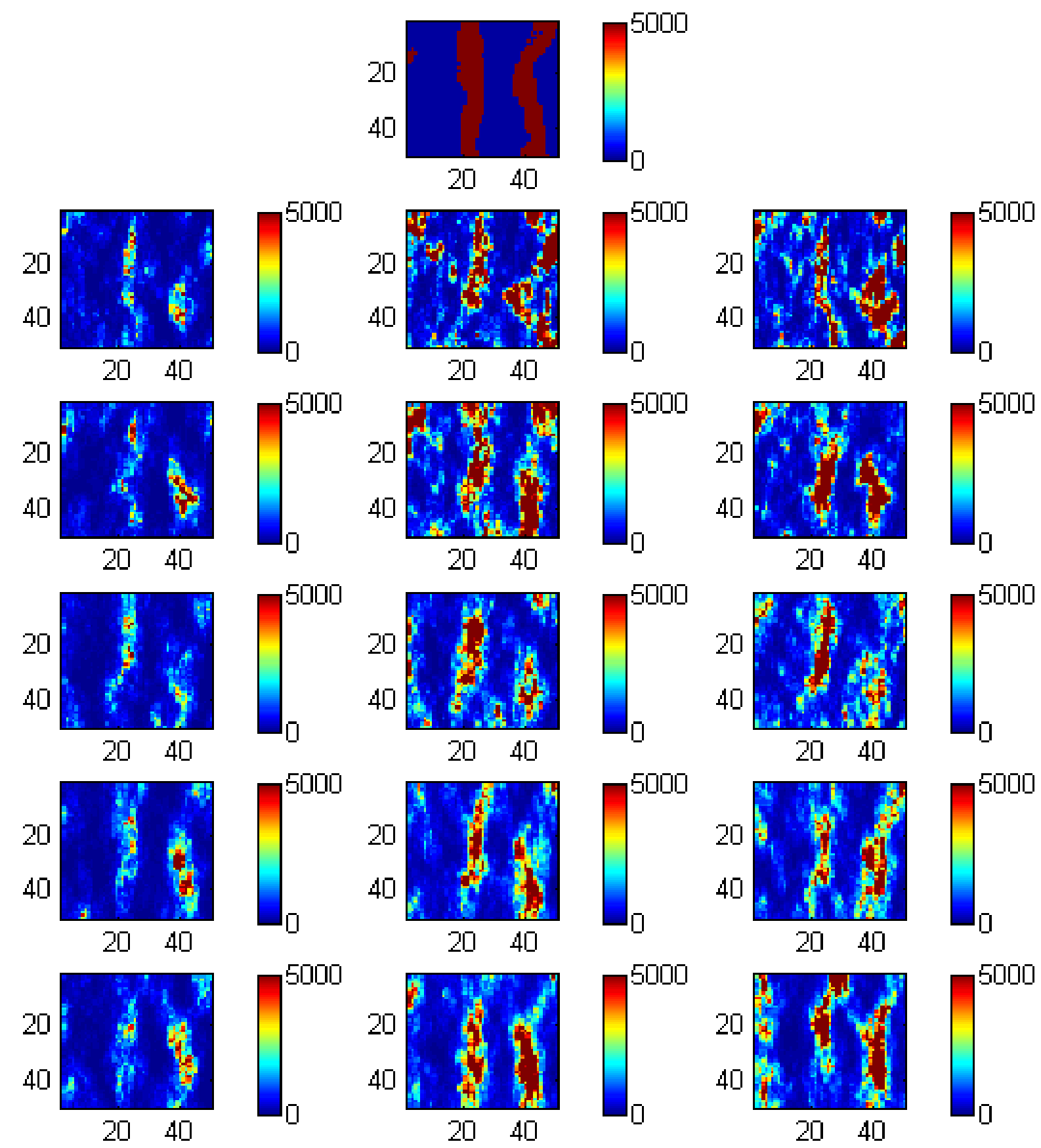

Figure 7: 1st Row: Reference Permeability field, Rows 2 to 6: Recovered fields at the end of 5-years history matching period with an ensemble size of 40,80,120, 160 and 200 respectively with the Standard EnKF (left column), SGSD-EnKF with OMP (middle column), and SGSD-EnKF with SABMP (right column). All values and scales are in units of millidarcy. The filters are implemented using $20 \%$ basis elements from the K-SVD dictionary for the SGSD-EnKF. 


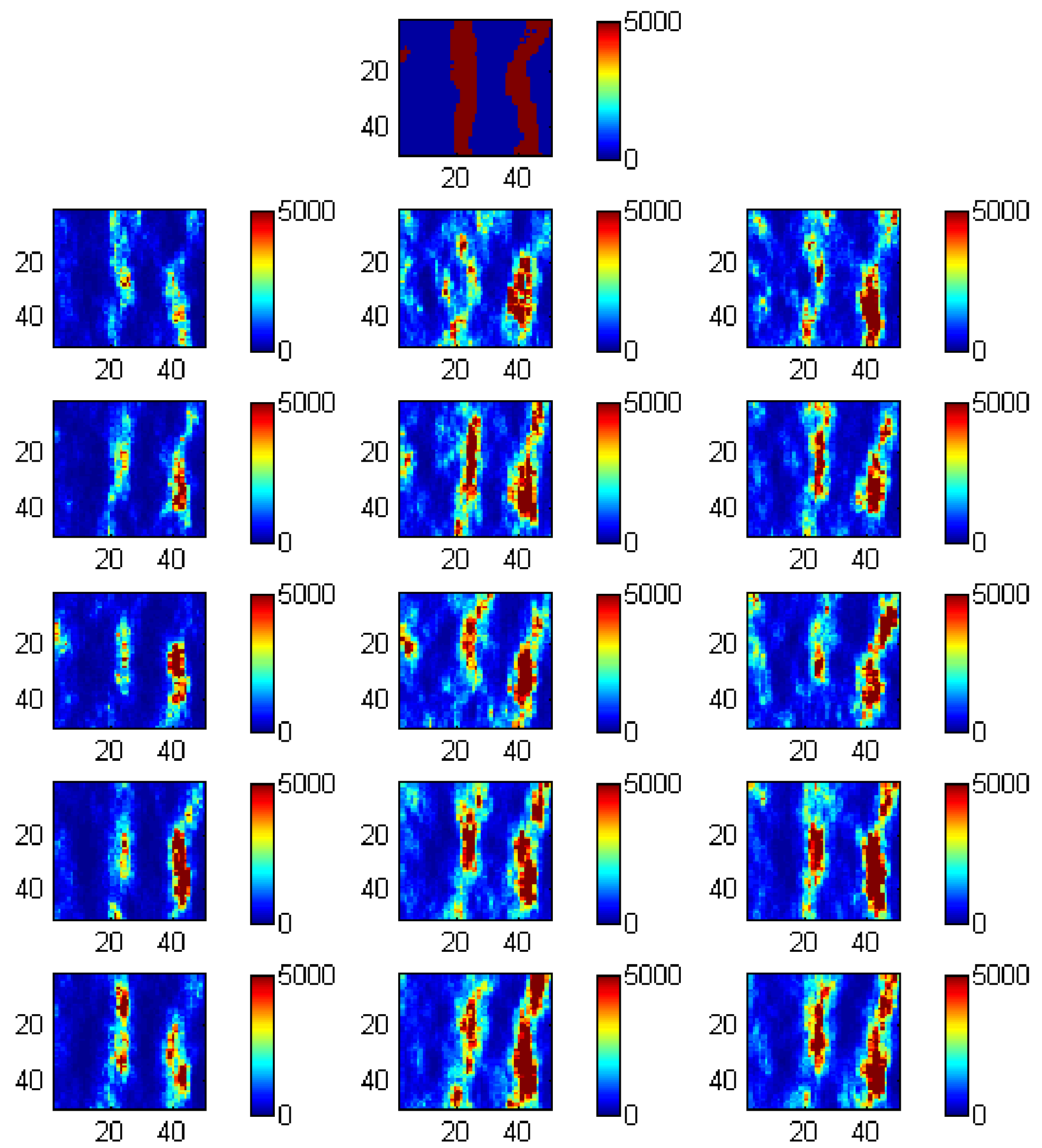

Figure 8: 1st Row: Reference Permeability field, Rows 2 to 6: Recovered fields at the end of 5-years history matching period with an ensemble size of 240, 280,320, 360 and 400 respectively with the Standard EnKF (left column), SGSD-EnKF with OMP (middle column), and SGSD-EnKF with SABMP (right column). All values and scales are in units of millidarcy. The filters are implemented using $20 \%$ basis elements from the K-SVD dictionary for the SGSD-EnKF. 


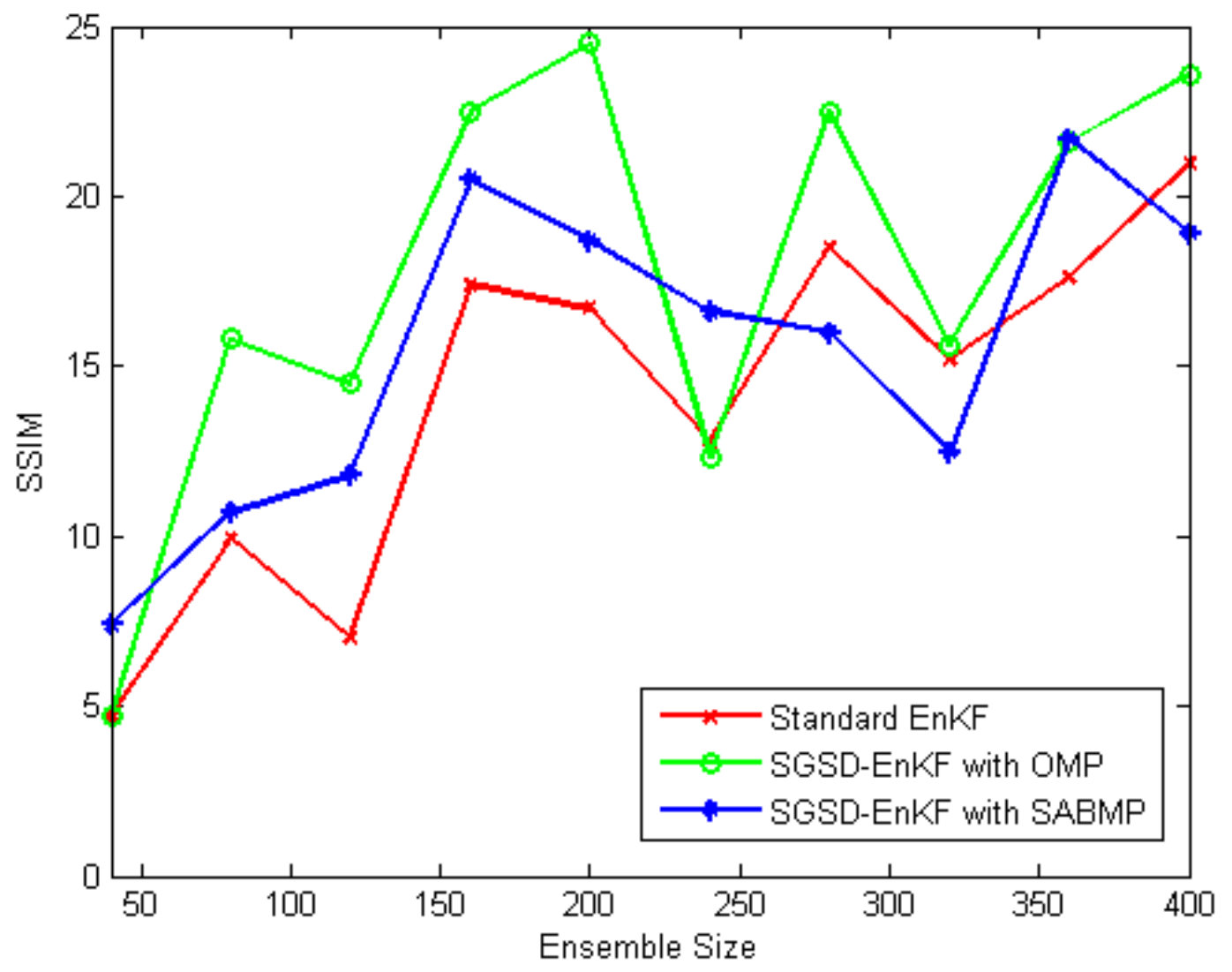

Figure 9: Estimation quality of the recovered permeability field, in terms of the SSIM, against increasing ensembles sizes for the experiment shown in Figure 7 and Figure 8. 


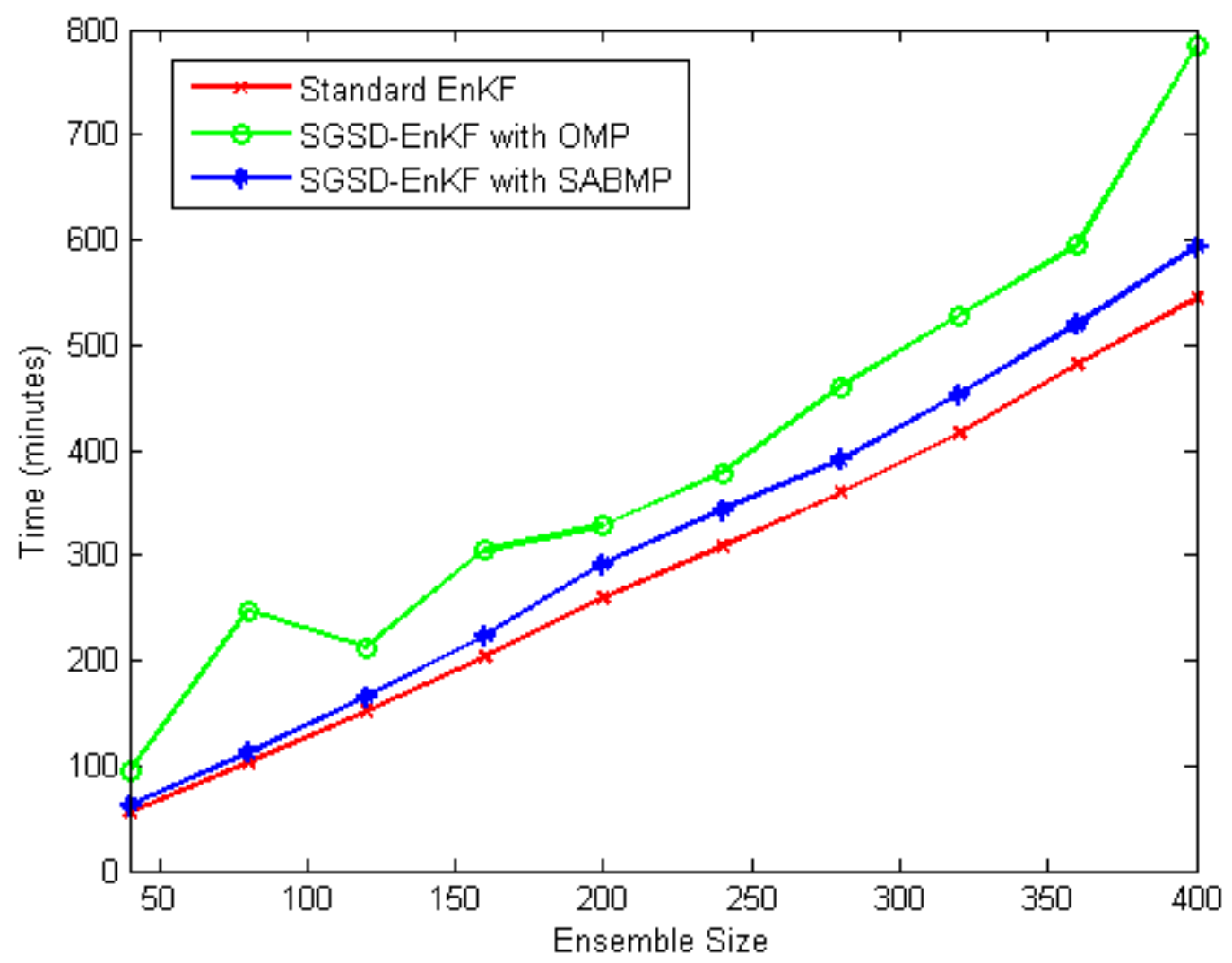

Figure 10: Analysis of computational times against increasing ensembles sizes for the experiment shown in Figure 7 and Figure 8. 


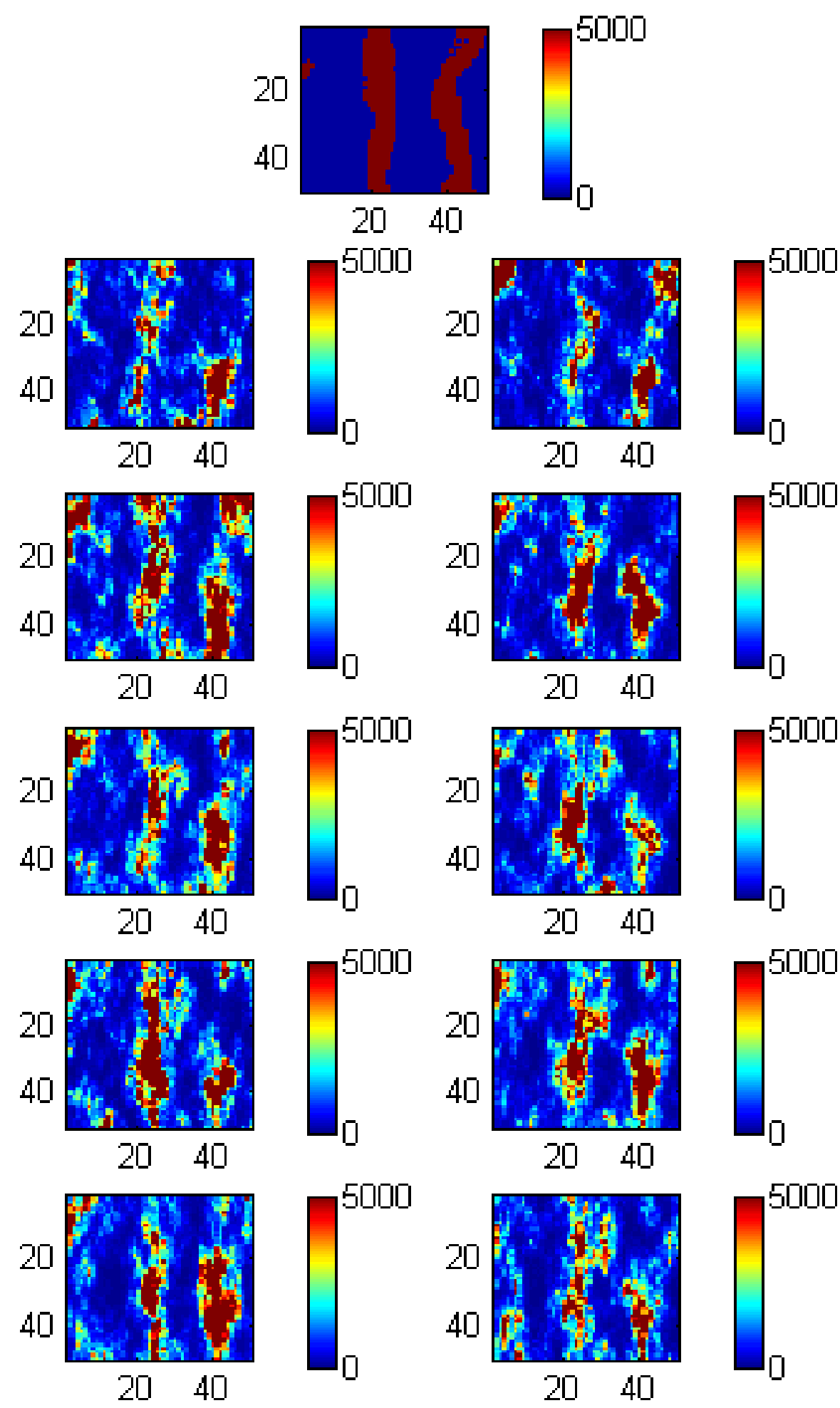

46

Figure 11: 1st Row: Reference Permeability field, Rows 2 to 6: Recovered fields at the end of 5-years history matching period with sparsity rates of $10 \%, 20 \%, 30 \%, 40 \%$ and $50 \%$ respectively using SGSD-EnKF with OMP (left column), and SGSD-EnKF with SABMP (right column). All values and scales are in units of millidarcy. All filters are implemented with an ensemble of 80 members. 


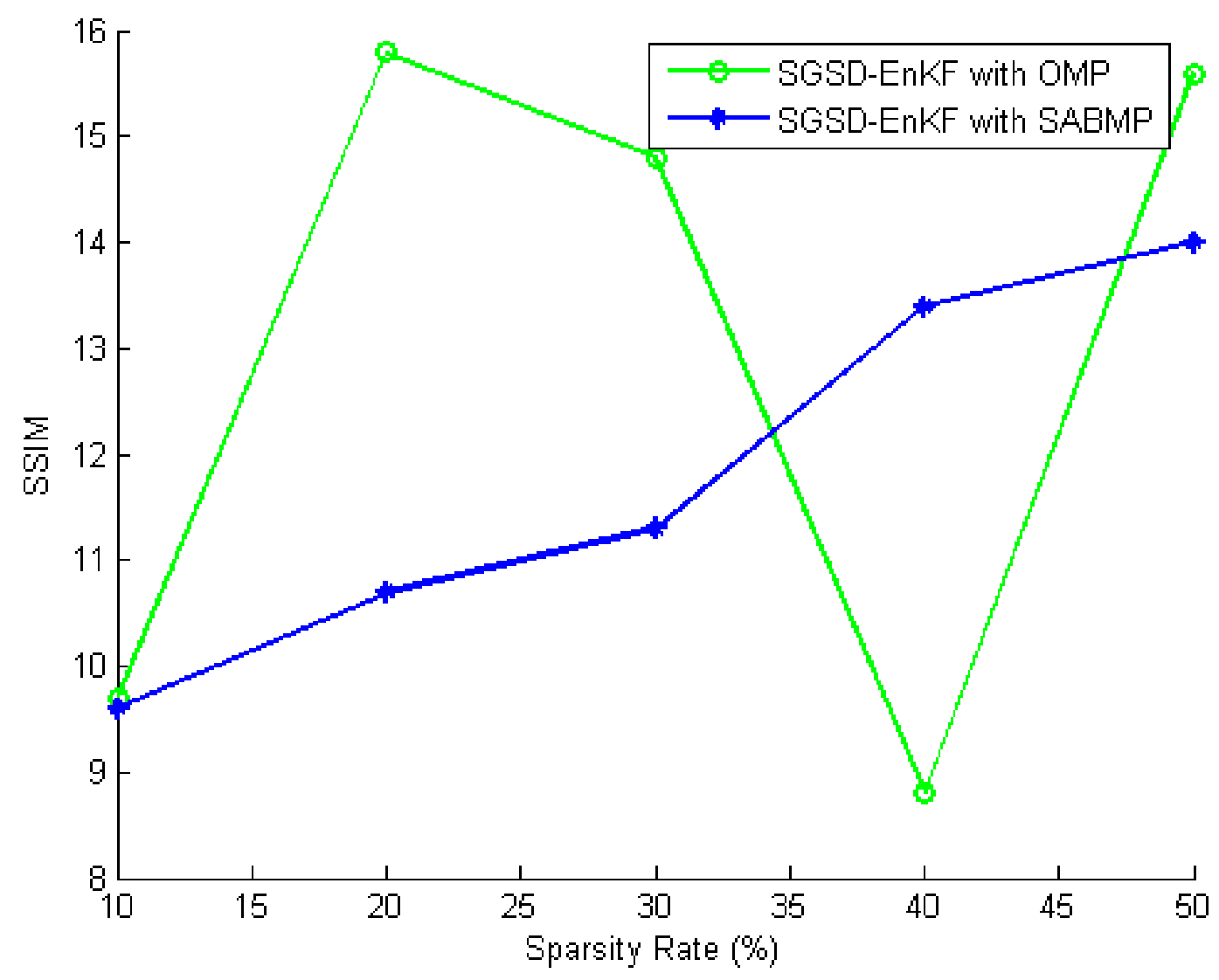

Figure 12: Estimation quality of the recovered permeability field, in terms of the SSIM, against increasing sparsity rates for the experiment shown in Figure 11. 


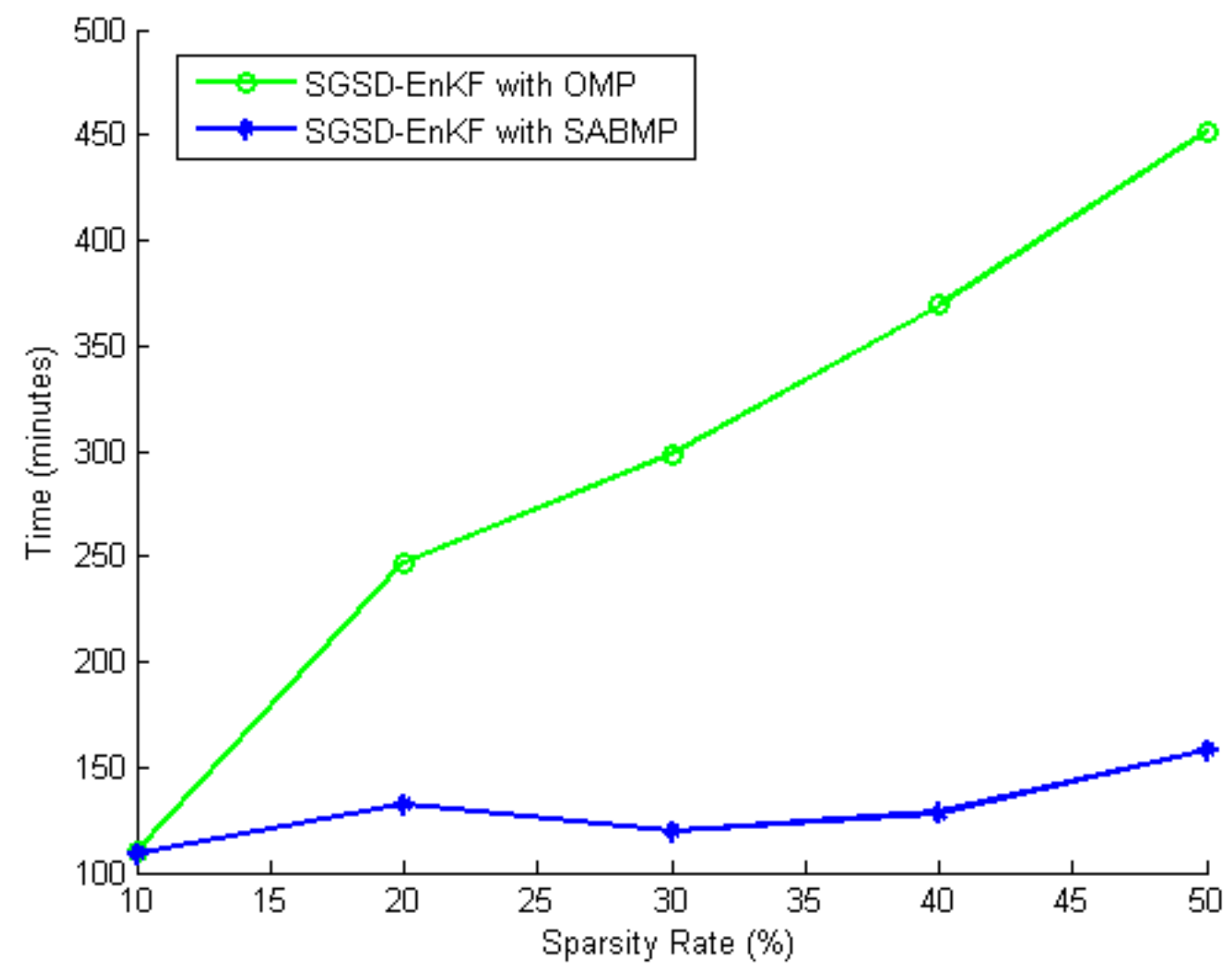

Figure 13: Analysis of computational times against increasing sparsity rates for the experiment shown in Figure 11. 